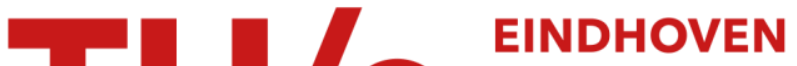 UNIVERSITY OF TECHNOLOGY
}

\section{Occupant behavior in building energy simulation: towards a fit- for-purpose modeling strategy}

Citation for published version (APA):

Gaetani , I., Hoes, P., \& Hensen, J. L. M. (2016). Occupant behavior in building energy simulation: towards a fitfor-purpose modeling strategy. Energy and Buildings, 121, 188-204.

https://doi.org/10.1016/j.enbuild.2016.03.038

\section{Document license:}

TAVERNE

DOI:

10.1016/j.enbuild.2016.03.038

Document status and date:

Published: 17/03/2016

\section{Document Version:}

Publisher's PDF, also known as Version of Record (includes final page, issue and volume numbers)

\section{Please check the document version of this publication:}

- A submitted manuscript is the version of the article upon submission and before peer-review. There can be important differences between the submitted version and the official published version of record. People interested in the research are advised to contact the author for the final version of the publication, or visit the $\mathrm{DOI}$ to the publisher's website.

- The final author version and the galley proof are versions of the publication after peer review.

- The final published version features the final layout of the paper including the volume, issue and page numbers.

Link to publication

\section{General rights}

Copyright and moral rights for the publications made accessible in the public portal are retained by the authors and/or other copyright owners and it is a condition of accessing publications that users recognise and abide by the legal requirements associated with these rights.

- Users may download and print one copy of any publication from the public portal for the purpose of private study or research.

- You may not further distribute the material or use it for any profit-making activity or commercial gain

- You may freely distribute the URL identifying the publication in the public portal.

If the publication is distributed under the terms of Article 25fa of the Dutch Copyright Act, indicated by the "Taverne" license above, please follow below link for the End User Agreement:

www.tue.nl/taverne

Take down policy

If you believe that this document breaches copyright please contact us at:

openaccess@tue.nl

providing details and we will investigate your claim. 


\title{
Occupant behavior in building energy simulation: Towards a fit-for-purpose modeling strategy
}

\author{
Isabella Gaetani*, Pieter-Jan Hoes, Jan L.M. Hensen \\ Building Physics and Services, Eindhoven University of Technology, P.O. Box 513, 5600 MB Eindhoven, The Netherlands
}

\section{A R T I C L E I N F O}

\section{Article history:}

Received 12 October 2015

Received in revised form 15 March 2016

Accepted 16 March 2016

Available online 17 March 2016

\section{Keywords:}

Occupant behavior modeling

Model complexity

Fit-for-purpose

Parsimony

\begin{abstract}
A B S T R A C T
Occupant behavior is nowadays acknowledged as a main source of discrepancy between predicted and actual building performance; therefore, researchers attempt to model occupants' presence and adaptive actions more realistically. Literature shows a proliferation of increasingly complex, data-based models that well fit the cases analyzed. However, the actual use of these models by practitioners is very limited. Moreover, simpler models might be preferable, depending on the aim of investigation. The present study proposes shifting the focus to fit-for-purpose modeling, in which the most appropriate model for a specific case is characterized by the lowest complexity, while preserving its validity with respect to the aim of the simulation. A number of steps are taken to achieve this shift in focus. The existing models are presented according to complexity. The available inter-comparison studies are critically reviewed. Subsequently, a list of parameters that affect the choice of an appropriate modeling strategy is presented as a first attempt to derive guidelines and generate a framework for investigation. To support such claims the effect of some of the listed parameters is evaluated in a case study. The main conclusion to be drawn is that determining the best complexity for occupant behavior modeling is strongly case specific.
\end{abstract}

(c) 2016 Elsevier B.V. All rights reserved.

\section{Introduction}

Occupants are responsible for a large share of a building's energy performance. As building envelopes and systems are optimized, technical performance standards become tighter and low-energy systems become widespread, the influence of occupant behavior on buildings' performance increases [1,2]. This influence can cause large deviations between predicted and actual performance, ultimately leading to a failure in achieving the desired building performance.

Two possible solutions to confront this problem are: improving performance predictions and changing the way occupants behave. As building scientists, our efforts typically concern the former strategy. Building energy simulations (BES) are a useful design-support tool, especially when it comes to comparing the performance of a range of designs or systems' concepts. However, when predicting the absolute energy performance of buildings such tools are still subjected to great uncertainty. This fact led ASHRAE [3] to state that neither the proposed building performance nor the baseline building performance represent actual energy consumption after

\footnotetext{
* Corresponding author.

E-mail address: i.i.gaetani@tue.nl (I. Gaetani).
}

construction. The first items from the listed sources of uncertainty are occupancy and building operation. Fig. 1 shows how researchers are increasingly concerned with the topic of occupant behavior in buildings.

A main issue with the introduction of occupant behavior models in building energy simulation (BES) is the choice of an appropriate modeling complexity for a specific case. In this paper complexity is defined, following [5], as the amount of detail in a model, which in turn depends on its size and resolution. Size refers to the number of components in a coupled model, while resolution refers to the number of variables in the model and their precision or granularity. The problem of selecting the right model complexity does not specifically concern building energy simulation, but rather simulation in general, which has led some researchers to state that "the choice of the best model is more of an art than a science" [6]. One of the pillars of modeling is the acknowledgement of the objectives of the model [7]: poorly understood modeling objectives can result in excessively complex models. This may cause errors in the simulation results, as well as unnecessary expenditure of time and money. Many studies (e.g. [8,9]) advocate the use of parsimonious models, i.e., the simplest among competing models. Generally, as simple models introduce approximation errors and complex models introduce uncertainty due to estimation, the goal of the simulation user should be to minimize the overall potential 


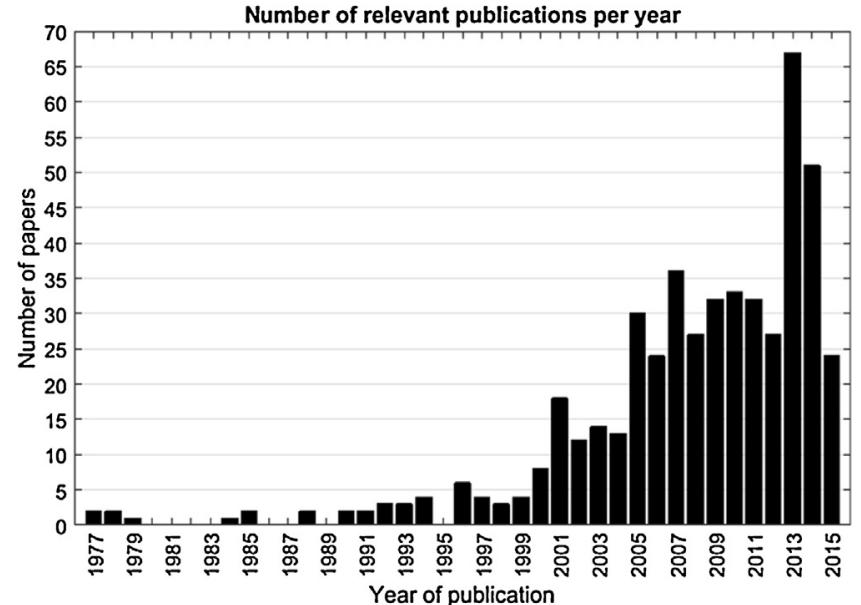

Fig. 1. Publications related to occupant behavior modeling and simulation as in Annex66 Occupants Behavior Research Bibliography [4] as of February 2016.

error by finding a compromise solution [10]. The optimal predictive ability of a model is nevertheless very case specific. Underfitting and overfitting occur when the model selection moves from the optimum towards too simple or too complex models, respectively (Fig. 2). It has to be noted that the resulting potential error from underfitting and overfitting may be comparable, depending on how far from the optimum a model is; however, in the case of overfitting additional time and cost efforts have to be taken into account.

In opposition to the principle of parsimony, a general increase in model complexity is observed. According to [11] this trend can be explained as a consequence of the following key factors: an "include-all syndrome" that leads modelers to include all available information; the progress of computing power that enables timeefficient, complex simulation; and unclear simulation objectives.

In regard to building energy simulation, a similar problem existed in well-researched subdomains such as modeling of airflow [12], lighting [13], or systems [10]. However, for these subdomains, it is now widely acknowledged that different complexity levels should be used for different aims of simulation. In the area of occupant behavior modeling, possibly due to its relatively recent development, a similar common understanding has not yet been reached.

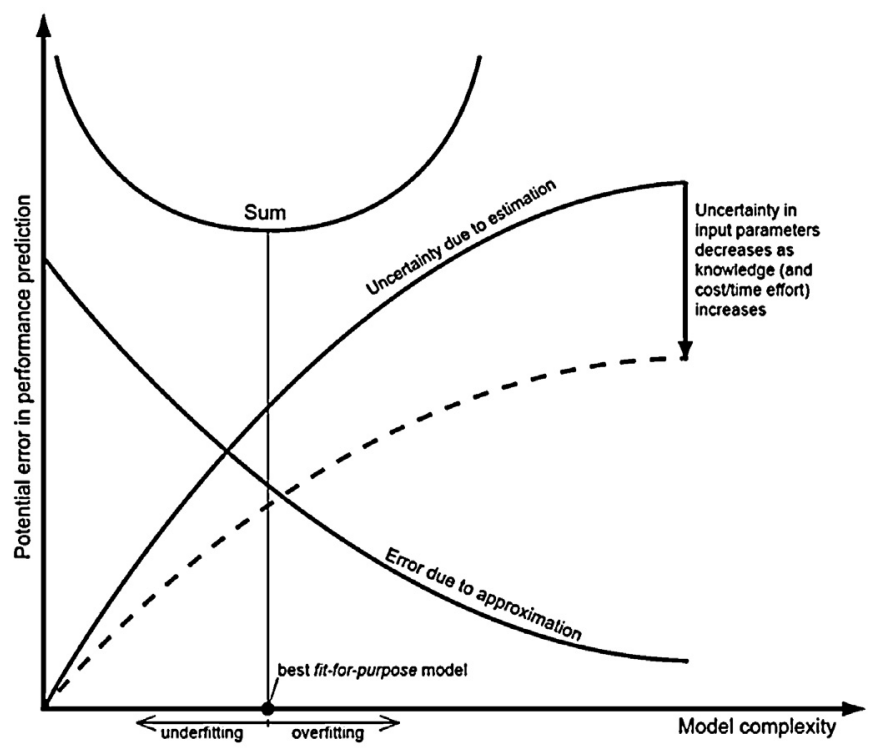

Fig. 2. Model uncertainty vs. complexity. Adapted from Trčka and Hensen [10].
The aim of this paper is to shape the dialogue concerning modeling complexity for occupant behavior in building energy simulation by investigating the merits of a fit-for-purpose approach. To accomplish this aim, the following questions are addressed: Which models are available? What is the difference between outcomes of these models? What is the most suitable model for a specific case (aim of investigation + building(s))? To answer these questions the current research presents an overview of the available models, provides a critical analysis of existing comparisons between models, and proposes a first draft of possible factors that could influence the choice of modeling technique. Our results suggest that future research efforts need to be directed at providing guidelines to select the most appropriate level of complexity in occupant behavior modeling. In addition, in order to definitively determine what happens to model predictions that are too complex or too simple, any potential decision method should be supported by practical demonstration.

\section{Overview of existing occupant behavior models}

Occupant behavior models are commonly divided into occupant movement and presence models, and action models. Action models comprise various types of (adaptive) behavior, such as adjusting shades and windows, switching on/off lights, using appliances, setting the thermostat, etc. A comprehensive occupant behavior model thus includes a series of sub-models.

In general, existing behavioral models can be classified according to their complexity [14,15]. Fixed, a priori schedules (also called diversity factors or profiles) represent the lowest level of complexity and are commonly implemented in BES software. Schedules are hourly fractions from 0 to 1 that act as multipliers of maximum quantities to define actual internal gains due to people, lighting loads, equipment loads, etc. Schedules are defined independently of the predicted conditions during the simulation. Hence, they represent a simplified scenario where the building operation is very predictable according to day-types. Schedules can either derive from standards or from observation-based statistically aggregated data. They can include deterministic rules, where actions are perceived as direct consequences of one or more drivers e.g., variation of indoor temperature or direct solar radiation. Given their deterministic nature, such models typically average out diversity of individuals, spaces/locations or time; therefore, they represent environments where the modeled behavior is always fully foreseeable and repeatable. While the size of the model is not directly affected, deterministic models add granularity by specifying various behavioral triggers, hence increasing the model resolution and the complexity. Data-based models are determined by the training profile and supposedly contain information about environmental triggers, but they cannot be referred to as deterministic models. We will refer to them as non-probabilistic models [16]. The main drawback of non-probabilistic models is their dependency on the dataset. Researchers have developed stochastic (or probabilistic) models to capture the variability of human behavior. In stochastic models, actions occur based on a probability function as a consequence of stimuli. Such models require a high number of runs to achieve reliable results [17], and cannot capture (e.g. time-) consistency. Also in this case, the size is not directly affected as the models still only consider the interactions between the occupants and the building. The resolution increases due to the multiple runs. Schedules, deterministic models, non-probabilistic models and probabilistic models represent the conventional simulation framework. A more complex simulation framework is defined by agent-based models, which switch from group-level to individual-level behavior predictions. Agent-based models predict the influence of occupants by modeling individuals, their mutual interactions and the interaction with the building. However, the 
Table 1

Overview of the most common occupant behavior modeling approaches according to size, resolution and complexity.

\begin{tabular}{lllll}
\hline $\begin{array}{l}\text { Simulation } \\
\text { framework }\end{array}$ & Type of model & Size & Resolution & Complexity \\
\hline Conventional & Schedules & $\bullet$ & $\bullet$ & $\bullet$ \\
& Deterministic & $\bullet$ & $\uparrow$ & $\uparrow$ \\
& Non-probabilistic & $\bullet$ & $\uparrow$ & $\uparrow$ \\
& Probabilistic/stochastic & $\bullet$ & $\uparrow \uparrow \uparrow$ & $\uparrow \uparrow \uparrow$ \\
Agent-based & Agent-based stochastic & $\uparrow \uparrow \uparrow$ & $\uparrow \uparrow \uparrow$ & $\uparrow \uparrow \uparrow \uparrow \uparrow \uparrow \uparrow \uparrow \uparrow$ \\
\hline
\end{tabular}

huge amount of information typically required (e.g., role of agents, relationship between agents, etc.) may not always be available. Despite this drawback, agent-based modeling currently commands the biggest share of publications in this niche [18]. Agent-based models markedly increase the size of the model as each individual is separately modeled; the resolution is still based on stochastic modeling and the resulting complexity is very high. Nonetheless, agent-based behavioral models can be characterized by different levels of complexity, depending on the complexity of the submodels which they include. Table 1 is a graphical representation of how modeling size, resolution and complexity are affected by changing the modeling strategy. The overall modeling complexity always derives from the complexity level of each sub-model. A detailed description of the available models follows.

Various reviews of existing occupant behavior models are available $[19,20]$. A first question to answer when performing a review is how to categorize the models. The most common categorizations are: according to complexity [14], according to object of investigation (occupancy or type of behavior) [21], and according to research approach [19]. Some authors have focused on one level of complexity or one object of investigation only, e.g. [18] on agent-based modeling and [21] on occupancy models. In the current research existing models have been classified according to complexity as the study deals with selecting the most appropriate level of modeling complexity. The focus of this literature review is on models that go beyond a priori schedules and simple deterministic rules. As briefly stated above, three main categories are identified: non-probabilistic models (defined as level "0"), which mainly include diversity factors resulting from data-mining; probabilistic or stochastic models (defined as level "1"), which represent the majority of the considered publications and rely on Logit analysis, Probit analysis, Markov chain processes, Poisson processes, and survival analysis [22]; agent-based and object-oriented models (defined as level "2"), also known as object-based models.

Table 2 provides an overview of the current status of occupant behavior modeling for building energy simulation. As well as the level of complexity (0-2) and the references for each model, seven other categories are specified, namely: model (M) or simulation framework (S); type of behavior; keywords; building typology; location; pros; cons. The distinction between model and simulation framework is made for practical purposes to underline whether particular attention has been placed on the integration of the model in BES or not. The type of behavior, building typology and location were identified as the main variables to take into account the case-specificity of each presented model. Keywords provide information about the modeling approach; the existing implementation in BES software; the validation status and the name of the model (where applicable). Lastly; pros and cons aim to highlight possible improvements to previous models; limitations to overcome; and general features and capabilities.

It has to be noted that this list does not claim to be exhaustive since new models are constantly being developed. Nevertheless, it gives an impression of the complexity of the occupant behavior modeling research field, of some recurrent issues and of the vast number of aspects that ought to be considered.
Some conclusions can be drawn from Table 2: (i) many models are available; (ii) models are rarely developed as a simulation framework: the implementation in BES mostly takes place on a project-based level, without guidelines for future use or measures for public availability; (iii) models are generally developed for a specific type of adaptive behavior, but recently there has been an increase of models which address the whole spectrum of user behavior; (iv) households and offices are the most investigated building typologies, and a large share of the investigated offices are single occupancy; (v) models are developed for specific locations, which might undermine their generalizability to other locations; (vi) different models are characterized by different specific advantages although a number of recurrent limitations are reported, e.g. complexity, case-specificity, lack of validation, calibration, generalizability, heavy dependency on (outdated) time use surveys. The findings of this overview are in line with the barriers that IEAEBC Annex 66 (Definition and Simulation of Occupant Behavior in Buildings) aims to overcome [75]. Although effort is being made to overcome the lack of integration of OB models in BES software (e.g., [76]), these conclusions reveal the difficulty for a potential simulation user to choose the most suitable model for a specific case. It has to be noted that the vast amount of developed models reveals the vibrant nature of the research field, which is still seeking to push the methodological boundaries of OB modeling practice. Typically, only a few of the research models will eventually achieve practical applications. The predictions of models characterized by different complexity levels have seldom been compared, and the differences among such predictions currently represent a knowledge gap. The few existing examples of inter-comparisons among models of different complexities are the focus of Section 3.

\section{Inter-comparisons of models with different complexity levels}

Section 2 demonstrates that the existing models are difficult to compare, both because of their case-specific nature and because of the lack of standardized methods to report and compare results [77]. Nevertheless, some comparisons among different models are available. For example, existing stochastic models have been researched $[22,58]$ to test for specific behaviors in offices and dwellings in order to define the best probabilistic approach. In the current research only comparisons among models of different complexities are taken into account, which to the best knowledge of the authors represent a very small share of publications.

Mahdavi and Tahmasebi [16] evaluated the probabilistic occupancy models developed by Reinhart for Lightswitch-2002 [30] and by Page et al. [39] by comparison with a newly developed non-probabilistic model. The object of the investigation is eight workspaces (single-occupancy, semi-closed individual, open-plan area) in an office area of the Vienna University of Technology. Notably, the authors use separate sets of data to train and evaluate the models. Data are collected over nine months (November 2011-July 2012) with a 1.6 min time-step to generate 15 -min interval data. The predicted and actual occupancy profiles are compared by means of a 100-run Monte Carlo simulation using 4 statistics (first arrival, last departure, duration and transitions errors) for 90 working days between April 2012 and July 2012. The overall goal of the study is to support building systems controls. The results show that Reinhart's and Page et al.'s models perform quite similarly, with Reinhart's model offering slightly better prediction of first arrival and intermediate transitions. Overall, the predictive capability of all models was found to be low and none of them performed below the threshold error value considered acceptable by the authors. The non-probabilistic model is found to perform best The authors suggest that the random diversity in occupancy pat- 
Table 2

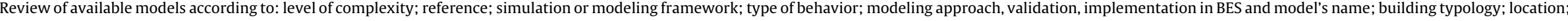
pros; cons.

\begin{tabular}{|c|c|c|c|c|c|c|c|c|}
\hline $\begin{array}{l}\text { Complexity } \\
(0=\text { non- } \\
\text { probabilistic, } \\
1=\text { probabilistic, } \\
2=\text { agent-based } \\
\text { models })\end{array}$ & Author(s) [Ref.] & $\begin{array}{l}\text { Model }(\mathrm{M}) \text { or } \\
\text { Simulation } \\
\text { framework (S) }\end{array}$ & Type of behavior & Keywords & Building Typology & Location & Pros & Cons \\
\hline 1 & Hunt [23] & M & Lighting control & $\begin{array}{l}\text { Probit analysis; } \\
\text { integrated ESP-r and } \\
\text { EnergyPlus }\end{array}$ & Office and school & UK & $\begin{array}{l}\text { Pioneer field-based stochastic } \\
\text { modeling; function of work } \\
\text { plane illuminance }\end{array}$ & $\begin{array}{l}\text { No combination with } \\
\text { other control laws (e.g. } \\
\text { dimming, occupancy } \\
\text { sensors) }\end{array}$ \\
\hline 1 & Fritsch et al. [24] & M & Windows opening & $\begin{array}{l}\text { Markov chain; } \\
\text { validated }\end{array}$ & Office & $\mathrm{CH}$ & $\begin{array}{l}\text { Window opening angle; } \\
\text { function of } \mathrm{T}_{\text {out }}\end{array}$ & $\begin{array}{l}\text { High dependence on } \\
\text { time series; winter } \\
\text { only }\end{array}$ \\
\hline 1 & Capasso et al. [25] & M & Load & $\begin{array}{l}\text { Monte Carlo; partially } \\
\text { validated }\end{array}$ & Household & I & $\begin{array}{l}\text { DSM application; accuracy; } \\
\text { aggregation and ownership }\end{array}$ & $\begin{array}{l}\text { High dependence on } \\
\text { outdated ('88-'89) } \\
\text { data; complexity of } \\
\text { input data }\end{array}$ \\
\hline 1 & Newsham et al. [26] & M & $\begin{array}{l}\text { Lighting } \\
\text { control + occupancy }\end{array}$ & $\begin{array}{l}\text { Markov chain; not } \\
\text { validated }\end{array}$ & Office & $\mathrm{CA}$ & $\begin{array}{l}\text { Function of work plane } \\
\text { illuminance; variety of lighting } \\
\text { controls }\end{array}$ & $\begin{array}{l}\text { Switch-on events } \\
\text { during occupation } \\
\text { period not considered }\end{array}$ \\
\hline 1 & Degelman [27] & M & Lighting control & $\begin{array}{l}\text { Monte Carlo; } \\
\text { integrated ENER-WIN }\end{array}$ & Office and university & US, JP & $\begin{array}{l}\text { Energy saving potential with } \\
\text { sensors/dimmers }\end{array}$ & $\begin{array}{l}\text { Not calibrated; no real } \\
\text { utility records }\end{array}$ \\
\hline 1 & Nicol [28] & M & $\begin{array}{l}\text { User behavior } \\
\text { (windows, lights, } \\
\text { blinds, heaters and fans } \\
\text { use) }\end{array}$ & Logit analysis & Office and household & F, GR, P, PAK, S, UK & $\begin{array}{l}\text { Function of } \mathrm{T}_{\text {out }} \text { and } \mathrm{T}_{\text {in }} ; \text { first } \\
\text { coherent prob. distribution for } \\
\text { windows' state }\end{array}$ & $\begin{array}{l}\text { Survey in form of } \\
\text { charts; no } \\
\text { consideration design, } \\
\text { systems etc. }\end{array}$ \\
\hline 1 & Yamaguchi et al. [29] & M & User behavior & Markov chain & Office & - & $\begin{array}{l}\text { Realistic presence/absence; } 4 \\
\text { working states; cogeneration; } \\
\text { costs and energy saving } \\
\text { strategies }\end{array}$ & $\begin{array}{l}2 \text { buildings' } \\
\text { combinations only; not } \\
\text { based on TUS; not clear } \\
\text { if whole year or } 1 \text { day; } \\
\text { long absences } \\
\text { neglected }\end{array}$ \\
\hline 1 & Reinhart [30] & M & $\begin{array}{l}\text { Lighting and blinds } \\
\text { control }\end{array}$ & $\begin{array}{l}\text { Inverse transform } \\
\text { sampling; integrated } \\
\text { Lightswitch Wizard, } \\
\text { DAYSIM, ESP-r and } \\
\text { EnergyPlus; } \\
\text { Ligtswitch2002 }\end{array}$ & Office & CA & $\begin{array}{l}\text { Function of occupancy/work } \\
\text { plane illuminance; } \\
\text { active/passive user; switching } \\
\text { actions depend on random } \\
\text { probability; blinds control } \\
\text { function of glare risk; improves } \\
\text { Newsham et al.'s model }\end{array}$ & $\begin{array}{l}\text { Fixed profiles } \\
\text { 7h45-18h15; presence } \\
\text { overestimation (no } \\
\text { absences apart from } \\
\text { break); no } \\
\text { intermediate } \\
\text { switch-off; blinds fully } \\
\text { open or closed; no } \\
\text { thermal considerations } \\
\text { for blinds activation }\end{array}$ \\
\hline 2 & Stokes et al. [31] & M & Lighting control & $\begin{array}{l}\text { Object-based; } \\
\text { validated }\end{array}$ & Household & UK & $\begin{array}{l}\text { Fine time-scale ( } 1 \mathrm{~min}) ; \\
\text { flexible model design } \\
\text { (single/multiple dwellings); } \\
\text { aggregated demands }\end{array}$ & $\begin{array}{l}\text { Old ('96-'97) TUS; } \\
\text { dependence on large } \\
\text { amount of input } \\
\text { data/assumptions; } \\
\text { complexity }\end{array}$ \\
\hline 1 & Pfafferott and Herkel [32] & M & User behavior & $\begin{array}{l}\text { Monte Carlo; } \\
\text { integrated ESP-r }\end{array}$ & Office & $\mathrm{DE}$ & $\begin{array}{l}\text { Considers passive cooling } \\
\text { specifically }\end{array}$ & $\begin{array}{l}\text { Adiabatic boundary } \\
\text { conditions between } \\
\text { rooms; data for } 42 \text { days }\end{array}$ \\
\hline 1 & Wang et al. [33] & M & Occupancy & $\begin{array}{l}\text { Non-homogeneous } \\
\text { Poisson process; } \\
\text { integrated EnergyPlus; } \\
\text { partially validated }\end{array}$ & Office & US & $\begin{array}{l}\text { Vacant intervals' distribution } \\
\text { (improves Reinhart's model); } \\
\text { time varying; matches with } \\
\text { observation }\end{array}$ & $\begin{array}{l}\text { Fixed profiles } \\
\text { weekends/weekdays; } \\
\text { total presence } \\
\text { overestimated; } \\
\text { intervals do not fit well }\end{array}$ \\
\hline
\end{tabular}


Table 2 (Continued)

\begin{tabular}{|c|c|c|c|c|c|c|c|c|}
\hline $\begin{array}{l}\text { Complexity } \\
(0=\text { non- } \\
\text { probabilistic, } \\
1=\text { probabilistic, } \\
2=\text { agent-based } \\
\text { models })\end{array}$ & Author(s) [Ref.] & $\begin{array}{l}\text { Model (M) or } \\
\text { Simulation } \\
\text { framework (S) }\end{array}$ & Type of behavior & Keywords & Building Typology & Location & Pros & Cons \\
\hline 2 & Bourgeois et al. [34] & $\mathrm{S}$ & $\begin{array}{l}\text { User behavior (lights, } \\
\text { blinds, windows and } \\
\text { equipment use) }\end{array}$ & $\begin{array}{l}\text { Object-based; } \\
\text { integrated ESP-r; } \\
\text { SHOCC }\end{array}$ & Office & CA, I & $\begin{array}{l}\text { Self-contained simulation } \\
\text { module; fully expandable; } \\
\text { improves Nicol's and } \\
\text { Reinhart's models }\end{array}$ & $\begin{array}{l}\text { No advanced solar } \\
\text { shading; lacks } \\
\text { flexibility; } \\
\text { deterministic } \\
\text { definition of passive } \\
\text { users }\end{array}$ \\
\hline 2 & Zimmermann [35] & M & User behavior & $\begin{array}{l}\text { Agent-based; } \\
\text { validated; integrated } \\
\text { BES }\end{array}$ & Office & US & $\begin{array}{l}\text { First agent-based application; } \\
\text { satisfactory fit; whole office } \\
\text { building }\end{array}$ & $\begin{array}{l}\text { Building/service/control } \\
\text { models need } \\
\text { refinement; activity } \\
\text { patterns need } \\
\text { validation; lacks } \\
\text { generalization }\end{array}$ \\
\hline 1 & Yun and Steemers [36] & M & Windows opening & $\begin{array}{l}\text { Logit analysis; } \\
\text { validated; integrated } \\
\text { ESP-r and EnergyPlus }\end{array}$ & Office & UK & $\begin{array}{l}\text { Function of } \mathrm{T}_{\text {in }} \text {, time of day, } \\
\text { previous window state; } \\
\text { separate sub-models for WO at } \\
\text { arrival, departure, during } \\
\text { occupancy }\end{array}$ & $\begin{array}{l}\text { Summer only; } 6 \text { offices } \\
\text { only; considers natural } \\
\text { ventilation behavior } \\
\text { but not exhaustively }\end{array}$ \\
\hline 2 & Tanimoto et al. [37] & M & User behavior & Agent-based; validated & Household & JP & $\begin{array}{l}\text { Pioneers of TUS data use; } \\
\text { considers electricity and DHW }\end{array}$ & \\
\hline 1 & Rijal et al. [38] & $\mathrm{s}$ & Windows opening & $\begin{array}{l}\text { Logit analysis; } \\
\text { validated; integrated } \\
\text { ESP-r and EnergyPlus }\end{array}$ & Office & UK & $\begin{array}{l}\text { Function of } \mathrm{T}_{\text {out }} \text { and } \mathrm{T}_{\text {in }} \text { (to } \\
\text { include building design); } \\
\text { active/passive user; develop } \\
\text { robust design solutions }\end{array}$ & $\begin{array}{l}\text { Old ('96-'97) TUS; not } \\
\text { clear how T } \mathrm{T}_{\text {out }} \text { is } \\
\text { considered; window } \\
\text { opening dead band } \\
\text { needs revision }\end{array}$ \\
\hline 1 & Page et al. [39] & $\mathrm{M}$ & Occupancy & $\begin{array}{l}\text { Markov chain } \\
\text { non-homogeneous } 2 \\
\text { states; validated; } \\
\text { MATLAB script; } \\
\text { integrated EnergyPlus }\end{array}$ & Office and Household & $\mathrm{CH}$ & $\begin{array}{l}\text { Vast range of applications } \\
\text { (given the right inputs); } \\
\text { includes long absences; } \\
\text { comprehensive; realistic }\end{array}$ & $\begin{array}{l}\text { Long-term monitoring; } \\
\text { complex input; } \\
\text { underestimates total } \\
\text { absence; calibration on } \\
5 \text { university offices }\end{array}$ \\
\hline 1 & Haldi and Robinson [40] & M & $\begin{array}{l}\text { User behavior } \\
\text { (windows, blinds, fans, } \\
\text { doors, cold drinks, } \\
\text { activity and clothing) }\end{array}$ & $\begin{array}{l}\text { Logit analysis; } \\
\text { integrated EnergyPlus }\end{array}$ & Office & $\mathrm{CH}$ & $\begin{array}{l}\text { Function of work plane, } \\
\text { outdoor illuminance and } \\
\text { occupancy; covers many } \\
\text { activities; } \\
\text { personal/environmental } \\
\text { adaptation }\end{array}$ & $\begin{array}{l}\text { No reversal of adaptive } \\
\text { behavior; function of } \\
\mathrm{T}_{\text {out }} \text { and } \mathrm{T}_{\text {in }} \text { only }\end{array}$ \\
\hline 1 & Herkel et al. [41] & $\mathrm{M}$ & Windows opening & $\begin{array}{l}2 \text { stochastic processes } \\
\text { for occupancy and } \\
\text { window opening; } \\
\text { sub-models arrival, } \\
\text { occupancy, departure }\end{array}$ & Office & $\mathrm{DE}$ & $\begin{array}{l}\text { Correlation with season; used } \\
\text { to assess robustness of natural } \\
\text { ventilation; most openings at } \\
\text { arrival }\end{array}$ & $\begin{array}{l}\text { Only predicts windows } \\
\text { status; lacks } \\
\text { generalization }\end{array}$ \\
\hline 1 & Richardson et al. [42] & $\mathrm{M}$ & $\begin{array}{l}\text { Lighting } \\
\text { control+occupancy }\end{array}$ & $\begin{array}{l}\text { Markov chain } \\
\text { non-homogeneous } 2 \\
\text { states MC; partially } \\
\text { validated; } \\
\text { implemented Excel }\end{array}$ & Household & UK & $\begin{array}{l}\text { Active/inactive users; sharing } \\
\text { behavior; combined with } \\
\text { activity model; free download }\end{array}$ & $\begin{array}{l}\text { Only classification } \\
\text { weekdays/weekends } \\
\text { and people/household }\end{array}$ \\
\hline 2 & Tanimoto et al. [42] & $\mathrm{M}$ & Load & Agent-based; validated & Household & $J P$ & $\begin{array}{l}\text { Public statistical data } \\
\text { (improves Page et al.'s model); } \\
\text { coupled with load calculation }\end{array}$ & $\begin{array}{l}\text { Complexity ( } 32 \\
\text { activities); only } \\
\text { summer, only cooling } \\
\text { loads; needs further } \\
\text { validation }\end{array}$ \\
\hline 1 & Widén et al. [44] & $\mathrm{M}$ & Activities & $\begin{array}{l}\text { Conversion of activity } \\
\text { data to energy load } \\
\text { profiled; validated; } \\
\text { MATLAB script }\end{array}$ & Household & $\mathrm{s}$ & $\begin{array}{l}\text { Realistic load distributions; } \\
\text { simplicity; considers various } \\
\text { household typologies }\end{array}$ & $\begin{array}{l}\text { Old ('96) TUS; heavy } \\
\text { dependence on TUS }\end{array}$ \\
\hline
\end{tabular}


Occupancy
Markov chain

non-homogeneous

Agent-based;

integrated eQuest

Artificial Neural

Network; integrate

TRNSYS; ESOM

Profiles derived by

validated

Bernoulli process based

on logit distribution,

Markov chain,

extension of Markov

chain to a

continuous-time

continuous-time
random process;

integration possible in

any BES; validated

Profiles derived by

data

Markov chain MC

validated

Household

Household

Office

Agent-based MC

Office

Markov chain

non-homogeneous MC

validated

Agent-based;

integrated eQuest

Agent-based;

validated; integrated

RADIANCE

Office

University

Offic
Absent/present

inactive/present active user

Optimize HVAC loads with occupancy-based energy

control

E

Comparison with Spanish

Technical Code for Buildings

(CTE); extensive data

CA Considers system performance

in cogeneration; typical

households function of

demand

$\mathrm{CH} \quad$ Extensive (7 yrs.)

measurements; function of

$\mathrm{T}_{\text {out }}, \mathrm{T}_{\text {in }}$, humidity, wind speed;

refinement active/passive;

extensive cross-validation
Clustering for weekday type; estimate of uncertainty (measurements)

Initial blind status,

indoor/outdoor illuminance input to Markov process:

separate sub-models for

chosen shaded fraction

Many activities; realistic events; whole office building

Balanced complexity/output quality; considers various household typologies

\section{Includes change over time by}

considering word-of-mouth

Specific design questions; GUI; effect on comfort
Old ('96) TUS;

problems in night-time demand data

collection; lighting

sharing not included

Poor fit of occupancy

estimation (20\% error,

but shown to have low impact); relatively low

possible savings

No comparison with

real data; overal

unclear

Limited available

information;

underestimation of

base-loads; does not

consider HVAC

No extensive

elaboration on BES

integration;

building-specific

calibration; windows

angle opening needs

refinement

Building-specific;

many info needed

high dependence o

Single configuration of

blinds

Very building specific

input; lacks

generalization

Only covers electricity

demand; limited TU

data

No extensive

description of used agent-based mode

similarity a
scenarios

Only effect of

window-size

considered; no effect on energy 


\begin{tabular}{|c|c|c|c|c|c|c|c|c|}
\hline $\begin{array}{l}\text { Complexity } \\
(0=\text { non- } \\
\text { probabilistic, } \\
1=\text { probabilistic, } \\
2=\text { agent-based } \\
\text { models })\end{array}$ & Author(s) [Ref.] & $\begin{array}{l}\text { Model (M) or } \\
\text { Simulation } \\
\text { framework (S) }\end{array}$ & Type of behavior & Keywords & Building Typology & Location & Pros & Cons \\
\hline 1 & Parys et al. [20] & S & User behavior & $\begin{array}{l}\text { Markov chain MC; } \\
\text { integrated TRNSYS } 6\end{array}$ & Office & B & $\begin{array}{l}\text { Holistic approach; improves } \\
\text { Bourgeois et al.'s model }\end{array}$ & $\begin{array}{l}\text { Built-in error from } \\
\text { choice of drivers (not } \\
\text { exhaustive); not } \\
\text { validated; modest } \\
\text { change in energy } \\
\text { consumption due to OB } \\
\text { (in contrast with other } \\
\text { studies) }\end{array}$ \\
\hline 2 & Robinson et al. [55] & S & User behavior & Agent-based & & $\mathrm{CH}$ & $\begin{array}{l}\text { Basis for future simulation at } \\
\text { various scales (from building to } \\
\text { urban) }\end{array}$ & $\begin{array}{l}\text { Huge amount of info } \\
\text { needed; not clear if } \\
\text { integrated; not clear if } \\
\text { fully developed }\end{array}$ \\
\hline 1 & Wang et al. [56] & M & Occupancy & $\begin{array}{l}\text { Markov chain } \\
\text { homogeneous; } \\
\text { partially validated; } \\
\text { MATLAB script }\end{array}$ & Office & - & $\begin{array}{l}\text { Produces non-synchronous } \\
\text { change of } O \text { in time/uneven } \\
\text { distribution of } O \text { in space; } \\
\text { simplicity, accuracy }\end{array}$ & $\begin{array}{l}\text { Probability functions } \\
\text { not time-dependent; } \\
\text { arithmetic speed } \\
\text { problem; simple } \\
\text { validation/calibration } \\
\text { missing }\end{array}$ \\
\hline 1 & Yamaguchi et al. [57] & M & User behavior & $\begin{array}{l}\text { Markov chain; } \\
\text { modified Tanimoto's } \\
\text { approach (Roulette } \\
\text { Selection) }\end{array}$ & Household & JP & $\begin{array}{l}\text { Considers weekday, Saturday, } \\
\text { holiday; electricity } \\
\text { consumption; } 27 \text { behaviors; } \\
\text { compare different modeling } \\
\text { approaches }\end{array}$ & $\begin{array}{l}\text { Model underestimates } \\
\text { changes in behavior; } \\
\text { unclear integration BES }\end{array}$ \\
\hline 1 & Schweiker et al. [58] & M & Windows opening & Markov chain & Household & $\mathrm{CH}, \mathrm{JP}$ & $\begin{array}{l}\text { Comparisons with Haldi and } \\
\text { Robinson } 2009 \text { and Rijal et al. } \\
2007\end{array}$ & $\begin{array}{l}\text { Issues with external } \\
\text { calibration; poor } \\
\text { window use prediction } \\
\text { of models calibrated } \\
\text { from Swiss data for } \\
\text { Japan }\end{array}$ \\
\hline 2 & Liao et al. [59] & M & Occupancy & Agent-based; validated & Office & US & $\begin{array}{l}\text { Relates with former state } \\
\text { [Feng]; parallel } \\
\text { low-complexity model based } \\
\text { on covariance graphical model } \\
\text { framework; improves Page's } \\
\text { model }\end{array}$ & $\begin{array}{l}\text { Much information in } \\
\text { an instance; high } \\
\text { complexity; not suited } \\
\text { for real-time } \\
\text { occupancy } \\
\text { estimations; no clear } \\
\text { integration }\end{array}$ \\
\hline 1 & Zhang and Barret [60] & $\mathrm{M}$ & Windows opening & Probit analysis & Office & UK & $\begin{array}{l}\text { Considers orientation; consider } \\
\text { non-office spaces in office } \\
\text { buildings }\end{array}$ & $\begin{array}{l}\text { No presence/absence } \\
\text { data; only one driver } \\
\mathrm{T}_{\text {out }}<20^{\circ} \mathrm{C} \text {; only one } \\
\text { building; no validation } \\
\text { nor integration in BES }\end{array}$ \\
\hline 0 & Duarte et al. [15] & $\mathrm{M}$ & Occupancy & $\begin{array}{l}\text { Descriptive statistics; } \\
\text { validated }\end{array}$ & Office & US & $\begin{array}{l}\text { Clustering per weekday and } \\
\text { month type; comparison with } \\
\text { ASHRAE guidelines and Page } \\
\text { et al.'s model }\end{array}$ & $\begin{array}{l}\text { Open plan offices not } \\
\text { accurately described; } \\
\text { comparison with Page } \\
\text { et al.'s model unclear }\end{array}$ \\
\hline 2 & Wilke et al. [61] & $\mathrm{M}$ & Activities + occupancy & $\begin{array}{l}\text { Logit analysis } \\
\text { multinomial; Markov } \\
\text { chain higher-order } \\
\text { pre-process; Weibull } \\
\text { distribution; validated }\end{array}$ & Household & $\mathrm{F}$ & $\begin{array}{l}\text { Assigns duration at the start of } \\
\text { new occupancy state; activities } \\
\text { function of week day, } 17 \\
\text { socio-demographic variables }\end{array}$ & $\begin{array}{l}\text { No simultaneous } \\
\text { activities; no } \\
\text { electrical/water } \\
\text { appliances }\end{array}$ \\
\hline
\end{tabular}


Cumulative and probability distribution function

$1 \quad$ Aerts et al. [64]

Occupancy

hierarchical clustering

Agent-based

Logit analysis; not

Calibrated realistic schedules; validated; integrated IDA Ice

Agent-based; integrated EnergyPlus

User behavior

User behavior

Rysanek and Choudhary [69]
Object-based; no

validated; integrate TRNSYS, EnergyPlus; tool DELORES

Logit analysis; integrated EnergyPlus
Office and Household

Office

Household

Office
Comparison with deterministic simulation (window model most impact on difference); coherent, general, extensible

Ease of use comparable/bette than deterministic approach: straight-forward

\section{Considers 4 groups of} dwellings: veruran and mechanically sentilated

extensive database (200

cubicle offices)

key variables transition probability and duration et al.'s; calibration for

download; low complexity Considers optimized behavio climate conditions

Extensive range of measured variables

Comparison with UNI/TS 1300 ; issue of system sizing

vercome conceptual problem that occupant actions are discrete events
3 states, 7 occupancy patterns:
Occupancy function o $\mathrm{CO}_{2}$ levels; only data Jan to Aug

Occupancy only

Strong dependence on TUS; occupancy only

1 agent; no validation: no calibration

No validation; some variables only monitored in some rooms

1 key user; data from 1 dwelling; no data for DHW and heating; case-specific; based on past history of consumption

No validation; not yet DHW, appliances, HVAC set points, interactions among agents

Stand-alone software (not clear influence of environment); time step $1 \mathrm{~h}$; no relation
lights/occupants; no clustering holiday periods; lighting method not established nor validated Probability of adaptive behavior always non-zero 
Table 2 (Continued)

\begin{tabular}{|c|c|c|c|c|c|c|c|c|}
\hline $\begin{array}{l}\text { Complexity } \\
(0=\text { non- } \\
\text { probabilistic, } \\
1=\text { probabilistic, } \\
2=\text { agent-based } \\
\text { models) }\end{array}$ & Author(s) [Ref.] & $\begin{array}{l}\text { Model (M) or } \\
\text { Simulation } \\
\text { framework (S) }\end{array}$ & Type of behavior & Keywords & Building Typology & Location & Pros & Cons \\
\hline 0 & Mahdavi and Tahmasebi [16] & M & Occupancy & $\begin{array}{l}\text { Statistically aggregated } \\
\text { profiles; building } \\
\text { systems control }\end{array}$ & Office & A & $\begin{array}{l}\text { Separate sets of data used for } \\
\text { training and evaluating the } \\
\text { models; comparison with } \\
\text { Reinhart's and Page et al.'s } \\
\text { models }\end{array}$ & $\begin{array}{l}\text { It returns the same } \\
\text { daily occupancy profile } \\
\text { for any given aggregate } \\
\text { profile of presence } \\
\text { probability used for } \\
\text { model training } \\
\text { (deterministic nature) }\end{array}$ \\
\hline 1 & D’Oca et al. [71] & M & $\begin{array}{l}\text { Windows } \\
\text { opening + thermostat }\end{array}$ & $\begin{array}{l}\text { Logit analysis; } \\
\text { integrated IDA Ice }\end{array}$ & Household & DK & $\begin{array}{l}\text { Active/medium/passive user; } \\
\text { compares } 5 \text { scenarios from } \\
\text { deterministic to probabilistic }\end{array}$ & $\begin{array}{l}\text { No comparison with } \\
\text { measured values; case } \\
\text { specific }\end{array}$ \\
\hline 2 & Langevin et al. [72] & $\mathrm{s}$ & User behavior & $\begin{array}{l}\text { Agent-based; } \\
\text { integrated EnergyPlus } \\
\text { with BCVTB; HABIT }\end{array}$ & Office & US & $\begin{array}{l}\text { Considers energy and thermal } \\
\text { comfort; multiple zones, offices }\end{array}$ & $\begin{array}{l}\text { Interaction between EP } \\
\text { simulation and } \\
\text { MATLAB behavior } \\
\text { model takes place at } \\
\text { every time-step; } \\
\text { multiple runs needed } \\
\text { (probabilistic nature) }\end{array}$ \\
\hline 2 & Alfakara and Croxford [73] & $\mathrm{s}$ & $\begin{array}{l}\text { Windows } \\
\text { opening + cooling }\end{array}$ & $\begin{array}{l}\text { Agent-based; } \\
\text { integrated TAS }\end{array}$ & Household & UK & $\begin{array}{l}\text { Response to summer } \\
\text { overheating }\end{array}$ & $\begin{array}{l}\text { Agents' actions } \\
\text { (lighting, windows, } \\
\text { cooling,...) fed to BS } \\
\text { model every time step; } \\
\text { only } 2 \text { occupants }\end{array}$ \\
\hline 1 & Zhou et al. [74] & M & Lighting control & $\begin{array}{l}\text { Poisson process; } \\
\text { validated for weekdays }\end{array}$ & Office & $\mathrm{CN}$ & $\begin{array}{l}\text { Considers time-varying nature } \\
\text { peak usage; uncertainties in } \\
\text { occupant behavior (main } \\
\text { driver) }\end{array}$ & $\begin{array}{l}\text { Case specific (large } \\
\text { offices with no daylight } \\
\text { control) }\end{array}$ \\
\hline 2 & Feng et al. [21] & $\mathrm{s}$ & Occupancy & $\begin{array}{l}\text { Object-based; } \\
\text { co-simulation for } \\
\text { integration }\end{array}$ & Office & - & $\begin{array}{l}\text { Various occupancy levels } \\
\text { integrated; flexible and } \\
\text { extensible, ease of updates or } \\
\text { maintenance }\end{array}$ & $\begin{array}{l}\text { Occupancy only; } \\
\text { complexity }\end{array}$ \\
\hline
\end{tabular}


terns reproduced by probabilistic models may be crucial for other aims of simulation (e.g. design and sizing of building systems), but they are not suitable to provide short-term occupancy predictions based on past data such as is needed in building systems control. In this case, non-probabilistic models that better fit historical data deliver a better predictive performance.

Tahmasebi et al. [78] apply occupancy, lighting and plug loads schedules from ASHRAE 90.1-2013 and Page et al.'s stochastic model [39] to a small-sized reference office model from the U.S. Department of Energy [79]. The aim of the study is to quantify the impact of stochasticity on annual and peak energy predictions for heating and cooling. The ASHRAE schedules were used as input for the stochastic model. The authors concluded that for the considered case the predictive ability of a simplified occupancy modeling approach is analogous to a more complex stochastic approach. In a follow-up study, Tahmasebi and Mahdavi [80] analyze the implications of using deterministic and stochastic presence models when predicting annual and peak energy demand for heating and cooling of a real office building in Vienna, Austria. The authors distinguish between the nature of the models (deterministic vs. stochastic), and also between the assumptions that were made while developing the model (generic assumptions vs. assumptions that rely on actual occupancy information). The results show that observation-based stochastic models have a better predictive ability of the internal heat gains if compared with fixed profiles. However, when specifically considering building-level energy performance indicators, it is the assumptions rather than the nature of the model which play a decisive role. Typically, standard-based assumptions overestimate the actual occupancy, resulting in higher cooling loads and lower heating loads.

Duarte et al. [15] evaluated new non-probabilistic occupancy diversity factors for private and open plan commercial office buildings against ASHRAE 90.12004 profiles and Page et al.'s model [39]. The schedules are based on data collected in 223 private offices over about two years (November 2009-October 2011) in a multitenant 11-story office building in Boise, Idaho. Although the overall trend throughout the day is similar, ASHRAE 90.12004 overestimated the occupancy level (peaks of $95 \%$ as opposed to about $50 \%$ predicted by the newly developed schedules for private offices). In addition, when comparing a typical high occupancy week and a typical low occupancy week with Page et al.'s model [39], the two models show similar characteristics, but the new data-based schedules do not register the great variation during the day found by the stochastic model. The authors hypothesize that this could be due to the restricted data set used to calibrate Page et al.'s model. Overall, the authors prove that the model resulting from measured data shows up to a $46 \%$ reduction in average day profile peaks for private office and about a $12 \%$ reduction for open plan office spaces when compared to the ASHRAE values. Interestingly, they point out that energy modelers could conduct two sets of simulations one with typical low and another with typical high occupancy profiles - in order to produce a range of expected energy consumption during the lifetime of the building, as opposed to a single value. This method would allow energy modelers not to unnecessarily complicate the model inputs.

D'Oca et al. [71] developed probabilistic user profiles for window opening and thermostat set-point adjustment. The yearly heating consumption obtained by implementing their profiles in IDA ICE is compared with the one from deterministic schedules of European standard EN 15251:2007. The new probabilistic model consists of logistic regression formulas and it is based on field measurements collected from January to August 2008 of 15 naturally ventilated dwellings near Copenhagen. The used time-step is $10 \mathrm{~min}$. The authors study 4 scenarios, namely: a deterministic scenario both for window opening and $\mathrm{T}_{\mathrm{sp}}$ that should function as a reference; $\mathrm{a}$ scenario where window opening is treated in a probabilistic way while $\mathrm{T}_{\mathrm{sp}}$ follows deterministic schedules; a probabilistic model for both behaviors; a probabilistic model which includes active, medium and passive users; a final model with 3 different thermostat adjustments. The three considered climates are: Athens, Stockholm and Frankfurt. When compared to the reference case, the last model showed the greatest discrepancy. Overall, it was pointed out how the deterministic approach generally underestimates the heating consumption, by the greatest magnitude in Athens (+61\%). The authors are confident that their findings can be applied during the whole-building lifecycle, namely: design phase, operation phase, building retrofit, building management and building codes and policy.

Langevin et al. [72] present the Human and Building Interaction Toolkit (HABIT), a co-simulation tool for comfort and behavior predictions based on a field-validated, agent-based scheme. The tool is developed in MATLAB and implemented in EnergyPlus by means of the Building Control Virtual Test Bed (BCVTB) middleware. The object of the investigation is a 3-story air-conditioned office building in Philadelphia, which was monitored for 1 year. The considered variables are: $\mathrm{T}_{\mathrm{sp} \text {,heating }}, \mathrm{T}_{\mathrm{sp}, \text { cooling, heater and fan equip- }}$ ment energy per person, clothing adjustment, fans/heaters, and window and thermostat adjustment. The various behaviors were grouped into three scenarios, namely: "Base", "Typical" and "Set Point Float" scenarios. The overall finding is that by considering a realistic behavior, expected energy use during winter in cold climates increases by up to $15 \%$. On the other hand, the authors point out that increasing the thermostat set points could counteract this rise and would significantly decrease the energy consumption in summer (up to $32 \%$ ).

Chapman et al. [68] propose a multi-agent simulation (MAS) approach to combine stochastic models into a single tool. This model-of-models integrates Page et al.'s presence model [39], Fanger's PMV model for metabolic gains calculation [81], Jaboob and Robinson's unpublished activities model, Haldi and Robinson's windows and shading devices model [22,50] and the Lightswitch2002 lighting algorithm [30]. The model is applied to a hypothetical house and a shoe box office; the results are compared with those obtained by means of default Design Builder schedules for each typology. The results for the residential building show a decrease from $68.7 \mathrm{kWh} / \mathrm{m}^{2}$ heating demand to $59.0 \mathrm{kWh} / \mathrm{m}^{2}$ ( $-16 \%)$ using the MAS model. The discrepancy increases to $-45 \%$ when considering the non-residential building $\left(91.0 \mathrm{kWh} / \mathrm{m}^{2}\right.$ and $62.4 \mathrm{kWh} / \mathrm{m}^{2}$ for schedules and MAS model, respectively). The window model represents the greatest contribution to the difference between results.

Azar and Menassa [53] take a slightly different approach when comparing the traditional eQuest building energy estimating model with an agent-based model. The authors consider three categories of occupants (high, medium and low energy consumers) according to blind position, lighting/equipment schedules and hot water consumption. The reference building is a $1000 \mathrm{sq}$. ft. graduate student room accommodating 10 students for over 60 months and is located in Madison, Wisconsin, US. The base case consists of all $10 \mathrm{stu}-$ dents belonging to the category "medium energy consumers". The authors use an agent-based model to simulate the effect of "word of mouth", presumably leading towards lower energy consumption. As a first step, the authors determined the share of electric and gas consumption directly influenced by occupants (79\% and $13 \%$, respectively). As far as it concerns electric use, the proposed method's consumption is - in the best-case-scenario - $21.6 \%$ lower than the eQuest average.

Yamaguchi et al. [57] developed two occupant behavior models based on the Monte Carlo approach (just as Richardson et al. [78] and Widén et al. [52] models) and on Tanimoto's [43] approach, and applied them to a household in Osaka, Japan. The modified version of Tanimoto's model is named Roulette Selection. The time-use 
Table 3

Considered comparison studies.

\begin{tabular}{|c|c|c|c|c|c|c|}
\hline \multirow[b]{2}{*}{ Author(s) [Ref.] } & \multirow[b]{2}{*}{ Type of behavior } & \multirow[b]{2}{*}{$\begin{array}{l}\text { Aim of simulation; performance } \\
\text { indicator; building typology }\end{array}$} & \multicolumn{4}{|c|}{$\begin{array}{l}\text { Models considered for comparison } \\
\sqrt{ }=\text { best performing } \operatorname{model}(\mathrm{s}) \times=\text { other considered } \operatorname{model}(\mathrm{s})\end{array}$} \\
\hline & & & Schedules & Non-probabilistic & Probabilistic & Agent-based \\
\hline $\begin{array}{l}\text { Mahdavi and } \\
\text { Tahmasebi [16] }\end{array}$ & Occupancy & $\begin{array}{l}\text { Systems control; daily occupancy } \\
\text { profile; ( single, semi-closed, } \\
\text { open-plan) office }\end{array}$ & & $\sqrt{ }$ & $x$ & \\
\hline Tahmasebi et al. [78] & $\begin{array}{l}\text { Occupancy, } \\
\text { lighting and } \\
\text { plug-loads }\end{array}$ & $\begin{array}{l}\text { Annual and peak energy demand for } \\
\text { heating and cooling; office }\end{array}$ & $\sqrt{ }$ & & $\sqrt{ }$ & \\
\hline $\begin{array}{l}\text { Tahmasebi and } \\
\text { Mahdavi [80] }\end{array}$ & Occupancy & $\begin{array}{l}\text { Annual and peak energy demand for } \\
\text { heating and cooling; office }\end{array}$ & $x$ & $\sqrt{ }$ (energy PIs) & $\begin{array}{l}\sqrt{ } \text { (presence } \\
\text { distribution and } \\
\text { peak values) }\end{array}$ & \\
\hline Duarte et al. [15] & Occupancy & $\begin{array}{l}\text { Daily occupancy profile; (single, } \\
\text { open-plan) office }\end{array}$ & $x$ & $\sqrt{ }$ & $x$ & \\
\hline D'Oca et al. [71] & $\begin{array}{l}\text { Window opening } \\
\text { and thermostat } \\
\text { adjustment }\end{array}$ & $\begin{array}{l}\text { Design; energy demand for heating; } \\
\text { household }\end{array}$ & $x$ & & $\sqrt{ }$ & \\
\hline Langevin et al. [72] & User behavior & $\begin{array}{l}\text { Energy demand and thermal } \\
\text { acceptability; office }\end{array}$ & $x$ & & & $\sqrt{ }$ \\
\hline Chapman et al. [68] & User behavior & $\begin{array}{l}\text { Design; energy demand; office and } \\
\text { household }\end{array}$ & $x$ & & & $\sqrt{ }$ \\
\hline Azar and Menassa [53] & $\begin{array}{l}\text { Blinds regulation, } \\
\text { lighting/ } \\
\text { equipment, DHW }\end{array}$ & Electric/gas demand; university & $x$ & & & $\sqrt{ }$ \\
\hline Yamaguchi et al. [57] & User behavior & $\begin{array}{l}\text { Behavior duration, start/end time, } \\
\text { number of transitions, probability } \\
\text { distribution, number of different } \\
\text { patterns }\end{array}$ & & & $\begin{array}{l}\sqrt{ } \text { (behavior } \\
\text { duration, } \\
\text { transitions) }\end{array}$ & $\begin{array}{l}\sqrt{ } \text { (variety of } \\
\text { behavior patterns) }\end{array}$ \\
\hline
\end{tabular}

data are collected during 9 days only. The results were evaluated in terms of: duration time for behaviors per day, time at which the routine behaviors start and end, number of behavior transitions per day, probability distribution showing percentage of behaviors at each time step, and number of different patterns of occupant behavior transition in 500 simulations. The authors conclude that the Markov Chain model well replicates behavior duration and transitions. However, the Roulette Selection better approximates the variety of behavior patterns, even with limited time use data, but it performs worse in terms of predictive capability of behavior transitions and duration.

Table 3 gives an overview of the considered comparisons. In most cases, such studies investigate differences between databased, newly developed models and standard profiles.

Other studies consider the issue of model complexity. However, their goal is not to compare the predictive performance of two or more models, hence they have not been included in Table 3 . Among them, Hong and Lin [2] investigate the effect of occupant behavior on a private office with three levels of complexity: direct use of EnergyPlus, use of the advanced Energy Management System in EnergyPlus, and use of modified code of EnergyPlus. The different complexities are used to model different aspects of occupant behavior. Liao et al. [59] acknowledge the need for different resolutions for different aims. For this reason, they propose the Multiple Modules (MuMo) model, a stochastic agent-based model for occupancy simulations over time, and a low-complexity occupancy model for real-time estimations. The MuMo model is shown to have a similar predictive capability to Page's model.

The comparisons presented in Table 3 are performed for different aims of simulation, performance indicators and building typologies (see Table 3). The fact that the publications are discordant on which models have a better predictive ability confirms that the capability of a model to predict reality strongly depends on both the considered case study and the performance indicators used. However, this observation is often implicit in the publications mentioned above, which tend to conclude - with some exceptions (e.g.,
[16]) - that a certain model simply has a better predictive ability (e.g., [71]). Moreover, some studies state that the models deemed to have a worse predictive performance overestimate the energy consumption, while other studies arrive at the opposite conclusion. Nonetheless, some conclusion can be drawn, i.e. that standard profiles are largely considered not to be suitable for describing complex occupant behaviors, that more complex models do not always perform best (e.g. in [16] a simple non-probabilistic model showed better convergence than a probabilistic one when compared to measured data), and that models derived by measured data always perform best when describing the investigated case study. However, such conclusions are of little help when facing a practical choice of which modeling complexity to use.

\section{The fit-for-purpose concept}

\subsection{Definition of fit-for-purpose}

How is it possible to determine which model performs best? Goodness-of-fit is considered to be an unsuitable method to compare models. Instead, a good fit is necessary but not sufficient, as many models are able to fit a dataset reasonably well without necessarily bearing any interpretable correlation with the underlying process. Generalizability to other datasets is proposed as a good measure of comparison [9]. The plain definition of fit-for-purpose is something good enough to do the job it was designed to do [83]. It is thus to be expected that different models were identified as having the best predictive capability for different aims, buildings, etc. Indeed, the simulation user should choose the model complexity according to the specific case. Previous studies indicate the need for different occupant behavior modeling techniques according to the aim of simulation [16,82,84-87], to the phase of the building lifecycle $[85,86,88]$, and to other building-related factors $[82,85,86]$. In particular, in [86] a conceptual multi-dimensional simulation deployment space is proposed. 


\subsection{The influential factors}

Table 4 shows a list of possible factors that could influence the choice of modeling technique. The intention should be to identify the simplest fit-for-purpose model, hence minimizing the potential prediction error. Typically, it will be possible to implement a simpler model in those cases where occupant behavior has a

Table 4

First draft of possible factors that could influence the choice of modeling technique divided in: object-related factors (a); aim of simulation (b); performance indicators (c); phase of building lifecycle (d).

\begin{tabular}{|c|c|}
\hline \multicolumn{2}{|l|}{ Object-related factors (a) } \\
\hline Building(s) function [89] & $\begin{array}{l}\text { Single family houses } \\
\text { Apartment blocks/multi-family houses } \\
\text { Offices } \\
\text { Educational buildings } \\
\text { Hospitals } \\
\text { Hotels and restaurants } \\
\text { Sports facilities } \\
\text { Wholesale and retail trade services } \\
\text { buildings } \\
\text { Other types of energy-consuming } \\
\text { buildings }\end{array}$ \\
\hline Building(s) characteristics & $\begin{array}{l}\text { Conditioned/living area }\left[\mathrm{m}^{2}\right] \\
\text { Conditioned volume }\left[\mathrm{m}^{3}\right] \\
\text { HVAC system concept } \\
\text { Ventilation strategy } \\
\text { Main orientation }\end{array}$ \\
\hline Interaction building/outdoor & $\begin{array}{l}\ldots \\
\text { S/V }\left[\mathrm{m}^{-1}\right] \\
\text { Windows area North facade [\%] } \\
\text { Windows area South facade [\%] } \\
\text { Windows area West facade [\%] } \\
\text { Windows area East facade [\%] } \\
\text { Glass type (U }\left[\mathrm{W} / \mathrm{m}^{2} \mathrm{~K}\right] \text {, SHGC [-], VT } \\
[-]) \\
\text { U value walls }\left[\mathrm{W} / \mathrm{m}^{2} \mathrm{~K}\right] \\
\mathrm{U} \text { value roof }\left[\mathrm{W} / \mathrm{m}^{2} \mathrm{~K}\right] \\
\mathrm{U} \text { value floor }\left[\mathrm{W} / \mathrm{m}^{2} \mathrm{~K}\right] \\
\text { Solar shading } \\
\text { Openable windows } \\
\text { Dynamic facades } \\
\text { Infiltration }\end{array}$ \\
\hline Interaction building/user & $\begin{array}{l}\text { Lighting control } \\
\text { Thermostat control } \\
\text { Windows control } \\
\text { Blinds control }\end{array}$ \\
\hline Climate characteristics & $\begin{array}{l}\cdots \\
\text { CDD } \\
\text { HDD } \\
\text { RH }\end{array}$ \\
\hline
\end{tabular}

Aim of simulation (b)

Policy making

Design

Retrofitting

Initial commissioning

On-going commissioning

Fault detection

Diagnostics

Control

...

Performance indicators (c)

Energy consumption
Table 4 (Continued)

\begin{tabular}{ll}
\hline Energy conservation & Avoided $\mathrm{CO}_{2}$ emissions $\left[\mathrm{kg} / \mathrm{m}^{2} \mathrm{y}\right]$ \\
& Savings (from $\mathrm{CO}_{2}$ and energy) [€/y] \\
& Operational costs [€/y] \\
& Max heating load [W] \\
& Max cooling load [W] \\
& Max lighting load [W] \\
& Max total load [W] \\
& Daylighting autonomy [\% hours not \\
& requiring electric lighting] \\
& Daylighting glare avoidance [\% hours in \\
& discomfort range (Daylighting Glare \\
Lighting & Index > =24, just uncomfortable)] \\
& Max T (a/op) in the zone [ $\left.{ }^{\circ} \mathrm{C}\right]$ \\
Visual comfort & Min T (a/op) in the zone [ $\left.{ }^{\circ} \mathrm{C}\right]$ \\
& \\
Thermal comfort & $\mathrm{PMV}[-]$ \\
& $\mathrm{PD}(\mathrm{predicted} \%$ dissatisfied due to \\
& draft $[\%]$
\end{tabular}

relatively lower impact on the performance indicator. Different building typologies have very different characteristic occupancy schedules that can be comparatively more or less predictable or constant throughout the day. For example, a school has a rather predictable occupancy schedule, while a dwelling is characterized by a much broader range of possibilities. When investigating the maximum heating load, a lower complexity than the one needed for the total heating energy consumption might be acceptable [80]. The selected performance indicator also influences the temporal granularity, or the choice of time-step during the energy simulation. In some phases of a building lifecycle e.g., design phase, it might not be possible to accurately predict the relevance of occupant behavior due to the lack of data or to the flexible design concept [88]. Finally, individual features of the building form, building envelope and building concept could also result in a variable influence of occupants. For example, in a museum users do not usually open windows, adjust blinds or the thermostat, while these are all basic requirements of acceptable comfort in households. The simple case study presented in Section 5 further demonstrates (i) how different typologies of behavior have a different impact on a given performance indicator, and(ii) how different performance indicators are differently influenced by occupant behavior. These results suggest that the most fit-for-purpose occupant behavior model might differ according to type of behavior as well as selected performance indicator. Our immediate future research will focus on identifying which of the factors or combination of factors presented in Table 4 and in Fig. 3 have most influence on the impact of occupant behavior on the predicted building energy and comfort performance.

\subsection{Fit-for-purpose framework}

A graphical representation of the line of thought that was followed in the research problem definition follows below. Fig. 3 represents how two different stakeholders might use building performance simulation for different aims, which in turn have an impact on the influential factors. The listed influential factors should primarily determine the choice of software and modeling approach for the overall energy simulation. Typically, the energy simulation model for a large district will have a lower complexity level than the one performed on a single building. The building modeling approach will itself influence the selection of a behavioral model. The influential factors will also play a role in the choice of occupant scenario, i.e. if the simulation should include a unique average occupant or a set of multiple occupant scenarios. The decision to use an occupant behavior model at all, and which specific model is selected, has important consequences on the evaluation of a building's energy performance [90]. This decision can result in great differences in the comparison of design alternatives, both on 


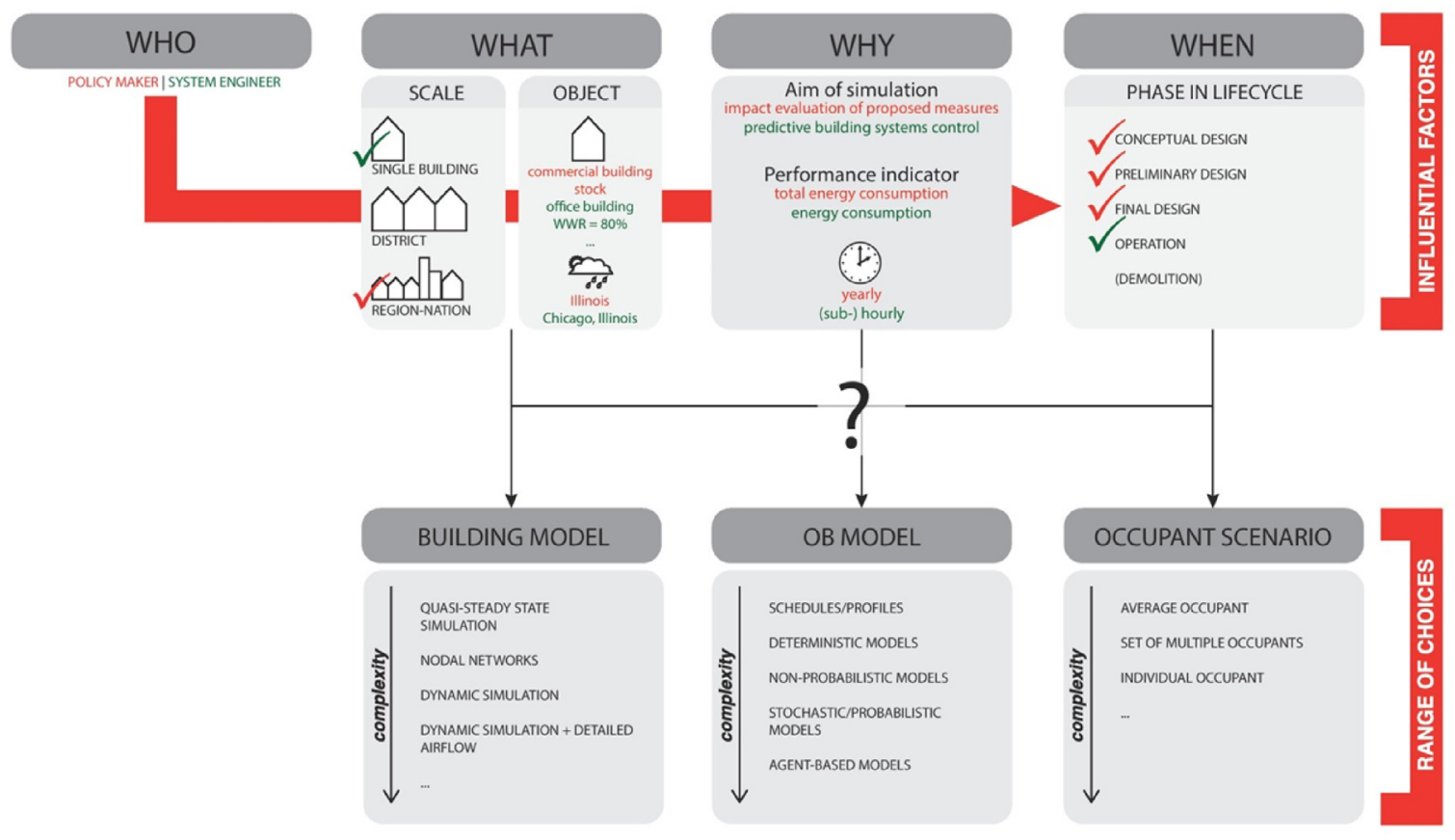

Fig. 3. Fit-for-purpose framework: policy maker vs. system engineer.

the building level, such as the percentage of glazing or shading [91], and with respect to system control [16], system sizing and a range of other choices.

\section{Case study}

As can be seen in Table 4, there are a large number of factors whose effects need to be determined. A small selection of these factors are investigated as a first demonstration of the fit-for-purpose approach in a simple case study. Future research by the current authors and others in the field will address these and other influential factors further.

\subsection{Reference building}

The medium-size office building model developed for EnergyPlus by the U.S. Department of Energy in the framework of commercial reference buildings [92] was selected as a case study. The reference building is a three-story office building located in Chicago, Illinois with a total floor area of $4982 \mathrm{~m}^{2}$. The building has an aspect ratio of 1.5 and a glazing fraction of 0.33 . The windows are non-operable and no sun screening system is present. Each floor of the building comprises one core zone and four perimeter zones. The roofs are flat and have insulation entirely above deck. The wall construction is steel frame. The wall construction,
Table 5

Building construction parameters.

\begin{tabular}{ll} 
Building construction & \\
\hline Window type & Double-pane window, low-e \\
& U-factor: $3.24 \mathrm{~W} / \mathrm{m}^{2} \mathrm{~K}$ \\
& SHGC: 0.385 \\
& VT: 0.305 \\
& Steel-Frame Walls \\
Wall construction & R-value: $1.95 \mathrm{~m}^{2} \mathrm{~K} / \mathrm{W}$ \\
& Built-up Roof: roof membrane + roof \\
Roof construction & insulation + metal decking \\
& R-value: $2.66 \mathrm{~m}^{2} \mathrm{~K} / \mathrm{W}$ \\
\hline
\end{tabular}

roof construction and window type of the reference buildings are location-dependent; Table 5 shows the construction of the considered reference building for Chicago, Illinois. The HVAC system consists of a furnace for heating, a packaged air-conditioning unit (PACU) for cooling and a multi-zone variable air volume (MZ VAV) for air distribution.

\subsection{Methodology}

The impact of occupant behavior on the building energy performance is assessed by introducing simple variations to the building operation. The selected operation parameters and their respective variations are illustrated in Table 6; reference values correspond to

Table 6

Operation parameters.

\begin{tabular}{|c|c|c|c|}
\hline Operation parameters & Low value & Reference & High value \\
\hline \multirow[t]{2}{*}{ HVAC Schedule } & Weekdays: 7am-6pm & Weekdays: 7am-10pm & Weekdays: 5am-12pm \\
\hline & Sat: $7 \mathrm{am}-6 \mathrm{pm}$ & Sat: $7 \mathrm{am}-6 \mathrm{pm}$ & Sat: 7am-6pm \\
\hline $\mathrm{T}_{\text {sp,heating }}\left[{ }^{\circ} \mathrm{C}\right]$ & 18 & 21 & 23 \\
\hline $\mathrm{T}_{\text {sp,cooling }}\left[{ }^{\circ} \mathrm{C}\right]$ & 22 & 24 & 26 \\
\hline Equipment Schedule & & See Fig. 4a & \\
\hline Equipment Power Density (EPD) $\left[\mathrm{W} / \mathrm{m}^{2}\right]$ & 5.38 & 10.76 & 16.14 \\
\hline Lights Schedule & & See Fig. $4 b$ & \\
\hline Lighting Power Density (LPD) [W/m²] & 5.38 & 10.76 & 16.14 \\
\hline Occupancy Schedule & & See Fig. 4c & \\
\hline Occupancy Rate [people/m²] & 0.027 & 0.054 & 0.107 \\
\hline
\end{tabular}


(a)
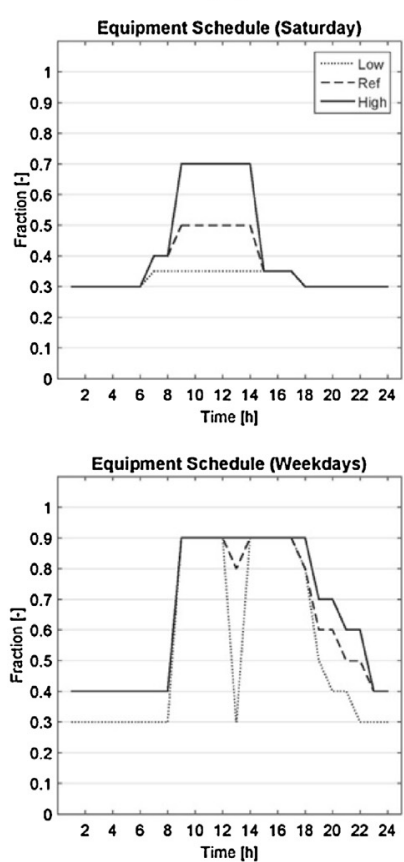

(b)

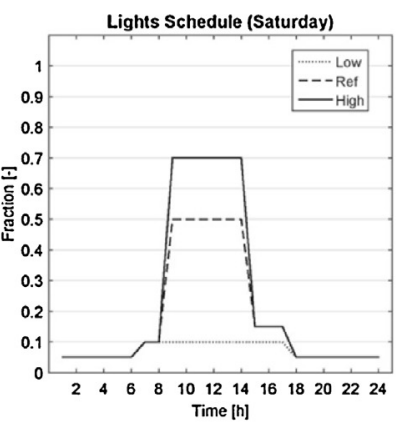

Lights Schedule (Weekdays)

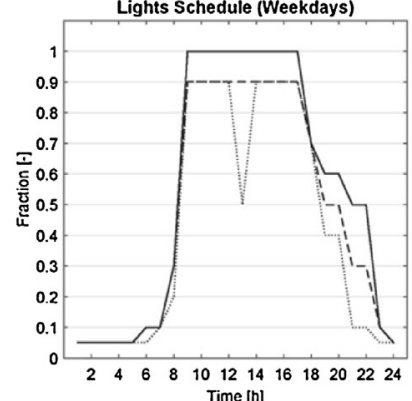

(c)
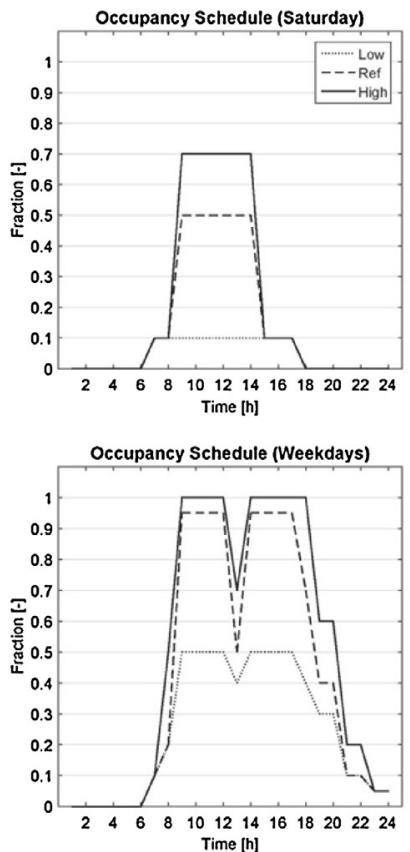

Fig. 4. Low, reference and high schedules for equipment use (a), lights use (b) and occupancy (c).

the original EnergyPlus model while most variations are as in [93]. Such operation parameters do not claim to represent all possible interactions of the occupants with a building, but are nevertheless a starting point for this research. The operation parameters are varied one-at-a-time to evaluate their individual impact on the considered performance indicator. Then, the combinations of parameters are investigated to study possible operation scenarios. Only the combinations of high and low values are considered in order to limit the number of simulations. Moreover, schedules for equipment use, light use and occupancy vary according to their corresponding value (e.g., at high equipment power density corresponds high equipment use schedule only), which leads to 64 scenarios in total. 16 of these scenarios are discarded as $\mathrm{T}_{\mathrm{sp} \text {, heating }}>\mathrm{T}_{\mathrm{sp} \text {,cooling; the }}$ remaining 48 scenarios are investigated.

The original building model uses the auto-sizing function of EnergyPlus to ensure that the system meets the peak loads. Thus, the size of the system would change for each scenario, as changing the operation parameters results in a modification of the Design-
Day values. In order to avoid this inaccuracy, which renders results impossible to compare, the system was sized for the reference scenario and kept constant throughout the simulations, as it would occur in reality (Fig. 4).

\subsection{Results and discussion}

Fig. 5 represents the percentage change of heating energy consumption and heating energy peak when varying each operation parameter, as well as the combinations of all parameters that show the highest (positive and negative) deviations in the results from the reference case. The results show how varying different operation parameters has a radically different effect on the selected performance indicator. For example, it is evident how the heating energy consumption is very much influenced only by

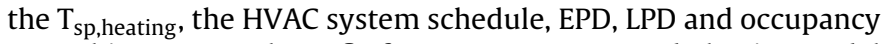
rate. This suggests that a fit-for-purpose occupant behavior model should be composed of sub-models characterized by different lev- (a)

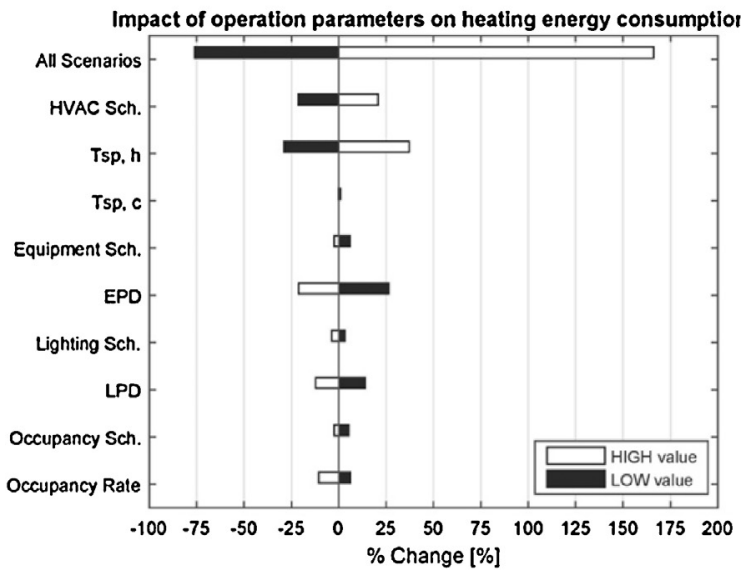

(b)

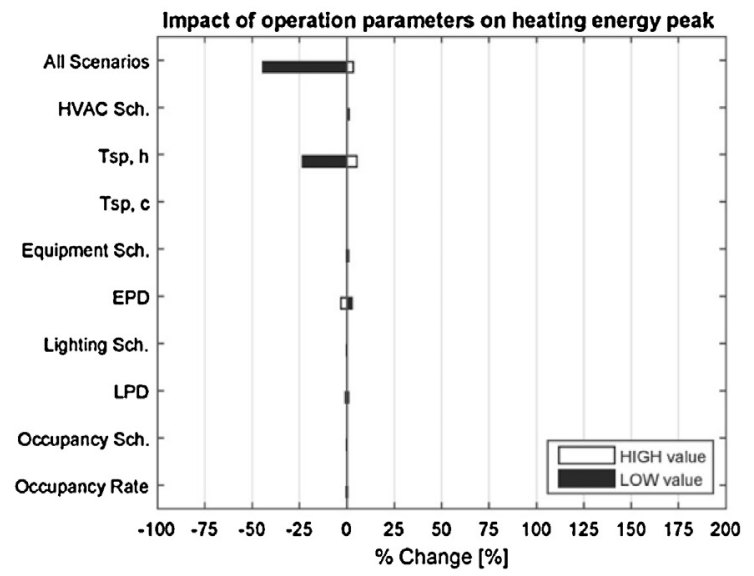

Fig. 5. Variations in heating energy consumption (a) and heating energy peak (b) due to operation scenarios. 
els of complexity. When looking at the heating energy peak, the only determining factors seem to be $\mathrm{T}_{\mathrm{sp} \text {,heating }}$ and EPD. Typically, the peak heating energy demand occurs at the first simulation time-step as soon as the HVAC system is activated to meet the temperature set-point (in the reference case, at 7 am daily), which might be the reason why parameters other than the $T_{\text {sp,heating }}$ have a negligible effect on the results. These results imply that - for the considered building and performance indicators - occupancy, lighting use and equipment use may be represented by simple schedules, as their effect on the energy performance is negligible. A higher level of complexity might be needed when representing other more influential operation parameters.

\section{Conclusions and further research}

Current practice in modeling occupant behavior is varied. A great number of increasingly complex models are being developed for many activities. However, the limited implementation of such models in BES software and the lack of inter-comparison between the outputs limits their use to researchers and developers rather than designers and engineers. To the best knowledge of the authors, there are no available guidelines to support the choice of a modeling technique with respect to occupant behavior according to aim of simulation, phase in the building lifecycle, type of building and other building(s)-related factors. Therefore, a switch in point of view, from the researcher to the simulation final user, is required to facilitate a fit-for-purpose modeling of occupant behavior in practice. By showing the very diverse conclusions of studies which compare existing models, this paper presents an attempt to focus on the impact of a range of modeling techniques on building performance assessment. A list of possible factors that might lead to a choice of modeling technique is presented. A simple case study illustrates how different aspects of occupant behavior influence a building's energy performance in a dissimilar way. Moreover, their impact changes according to the performance indicator under investigation. The principle of fit-for-purpose should be applied to choose which occupant behavior model is to be used when. Since the field is currently a hot topic of investigation, our research in the area is ongoing and many other researchers are making significant contributions to the field. Some of this research aims to support the initial effort to develop fit-for-purpose models and compare the outcomes of different modeling complexity for specific cases. The ultimate intention is to develop guidelines that could assist the final user of the simulation in selecting the most appropriate occupant behavior modeling technique for his/her specific case.

\section{Acknowledgements}

The support of PIT/VABI, the SPARK consortium and TKI EnerGO TRECO-office is greatly appreciated. This research was carried out as part of the IEA-EBC Annex 66 (Definition and Simulation of Occupant Behavior in Buildings).

\section{References}

[1] C.M. Clevenger, J. Haymaker, The impact of the building occupant on energy modeling simulations, in: Computing and Decision Making in Civil and Building Engineering, International Society for Computing in Civil and Building Engineering, Montreal, Canada, 2006.

[2] T. Hong, H. Lin, Occupant behavior: impact on energy use of private offices, Asim IBSPA Asia Conference (2012).

[3] ASHRAE, Addendum $r$ to ANSI/ASHRAE/IESNA Standard 90. 1-2007 Energy Standard for Buildings Except Low-Rise Residential Buildings (2007).

[4] IEA-EBC Annex 66, Occupants Behavior Research Bibliography, (n.d.) http:// www.annex66.org/?q=biblio.

[5] B.P. Zeigler, T.G. Kim, H. Praehofer, Theory of Modelling and Simulation, 2nd ed., Academic Press New York, New York, USA, 2000.
[6] R.J. Brooks, A.M. Tobias, Choosing the best model: level of detail, complexity, and model performance, Math. Comput. Modell. 24 (1996) 1-14, http://dx. doi.org/10.1016/0895-7177(96) 00103-3.

[7] S. Robinson, Choosing the right model: conceptual modeling for simulation, 2011 Winter Simulation Conference (2011) 1428-1440.

[8] R. Astrup, K.D. Coates, E. Hall, Finding the appropriate level of complexity for a simulation model: an example with a forest growth model, For. Ecol. Manage. 256 (2008) 1659-1665, http://dx.doi.org/10.1016/j.foreco.2008.07.016.

[9] M. a. Pitt, I.J. Myung, When a good fit can be Bad, Trends Cogn. Sci. 6 (2002) 421-425, http://dx.doi.org/10.1016/S1364-6613(02) 01964-2.

[10] M. Trčka, J.L.M. Hensen, Overview of HVAC system simulation, Autom. Constr. 19 (2010) 93-99, http://dx.doi.org/10.1016/j.autcon.2009.11.019.

[11] L. Chwif, M.R.P. Barretto, R.J. Paul, On simulation model complexity, 2000 Winter Simulation Conference Proceedings (Cat. No.00CH37165) (2000) 449-455, http://dx.doi.org/10.1109/WSC.2000.899751.

[12] E. Djunaedy, J.L.M. Hensen, M.G.L.C. Loomans, Toward external coupling of building energy and airflow modelling programs, ASHRAE Trans. (2003) 771-787.

[13] C.E. Ochoa, M.B.C. Aries, J.L.M. Hensen, State of the art in lighting simulation for building science: a literature review, J. Build. Perform. Simul. 5 (2012) 209-233, http://dx.doi.org/10.1080/19401493.2011.558211.

[14] H. Polinder, M. Schweiker, A. Van Der Aa, K. Schakib-Ekbatan, V. Fabi, R. Andersen, et al. Final Report Annex 53-Occupant behavior and modeling (Separate Document Volume II), 2013.

[15] C. Duarte, K. Van Den Wymelenberg, C. Rieger, Revealing occupancy patterns in an office building through the use of occupancy sensor data, Energy Build. 67 (2013) 587-595, http://dx.doi.org/10.1016/j.enbuild.2013.08.062.

[16] A. Mahdavi, F. Tahmasebi, Predicting people's presence in buildings: an empirically based model performance analysis, Energy Build. 86 (2015) 349-355, http://dx.doi.org/10.1016/j.enbuild.2014.10.027.

[17] J.A. Macdonald, Assessing uncertainty in building simulation, in: Proceedings of Building Simulation 99, IBPSA Conference, Kyoto, Japan, 1999, pp. 683-695.

[18] W. Parys, B. Souyri, M. Woloszyn, Agent-based behavioural models for residential buildings in dynamic building simulation: state-of-the-art and integrated model assembly, IBPSA-FR 2014 (2014) 1-8.

[19] H.B. Gunay, W. O’Brien, I. Beausoleil-Morrison, A critical review of observation studies, modeling, and simulation of adaptive occupant behaviors in offices, Build. Environ. 70 (2013) 31-47, http://dx.doi.org/10.1016/j. buildenv.2013.07.020.

[20] W. Parys, D. Saelens, H. Hens, Coupling of dynamic building simulation with stochastic modelling of occupant behaviour in offices-a review-based integrated methodology, J. Build. Perform. Simul. 4 (2011) 339-358, http://dx. doi.org/10.1080/19401493.2010.524711.

[21] X. Feng, D. Yan, T. Hong, Simulation of occupancy in buildings, Energy Build. 87 (2015) 348-359, http://dx.doi.org/10.1016/j.enbuild.2014.11.067.

[22] F. Haldi, D. Robinson, Interactions with window openings by office occupants, Build. Environ. 44 (2009) 2378-2395, http://dx.doi.org/10.1016/j.buildenv. 2009.03.025.

[23] D.R.G. Hunt, The use of artificial lighting in relation to daylight levels and occupancy, Build. Environ. 14 (1979) 21-33, http://dx.doi.org/10.1016/03601323(79) 90025-8.

[24] R. Fritsch, A. Kohler, M. Nygard-Ferguson, J.L. Scartezzini, A stochastic model of user behaviour regarding ventilation, Build. Environ. 25 (1990) 173-181, http://dx.doi.org/10.1016/0360-1323(90) 90030-U.

[25] A. Capasso, W. Grattieri, R. Lamedica, A. Prudenzi, Bottom-up approach to residential load modeling, IEEE Trans. Power Syst. 9 (1994) 957-964, http:// dx.doi.org/10.1109/59.317650.

[26] G.R. Newsham, Lightswitch: a stochastic model for predicting office lighting energy consumption, in: Proceedings of Right Light Three, 3rd European Conference on Energy Efficient Lighting, Newcastle, UK, 1995, pp. 59-66.

[27] L.O. Degelman, A model for simulation of daylighting and occupancy sensors as an energy control strategy for office buildings, in: Proceedings of Building Simulation 99, Kyoto, Japan, 1999, pp. 571-578.

[28] J.F. Nicol, Characterising occupant behavior in buildings: towards a stochastic model of occupant use of windows, lights, blinds heaters and fans, in: Seventh International IBPSA Conference, Rio de Janeiro, Brazil, 2001, pp. 1073-1078.

[29] Y. Yamaguchi, Development of district energy system simulation model based on detailed energy demand model, Proceeding of Eighth International IBPSA Conference (2003) 1443-1450.

[30] C.F. Reinhart, Lightswitch-2002: A model for manual and automated control of electric lighting and blinds, Sol. Energy 77 (2004) 15-28, http://dx.doi.org/ 10.1016/j.solener.2004.04.003.

[31] M. Stokes, M. Rylatt, K. Lomas, A simple model of domestic lighting demand, Energy Build. 36 (2004) 103-116, http://dx.doi.org/10.1016/j.enbuild.2003. 10.007.

[32] J. Pfafferott, S. Herkel, Statistical simulation of user behaviour in low-energy office buildings, International Conference Passive and Low Energy Cooling for the Built Environment (2005) 676-682, http://dx.doi.org/10.1016/j.solener. 2006.08.011.

[33] D. Wang, C.C. Federspiel, F. Rubinstein, Modeling occupancy in single Person offices, Energy Build. 37 (2005) 121-126, http://dx.doi.org/10.1016/j.enbuild. 2004.06.015.

[34] D. Bourgeois, C. Reinhart, I. Macdonald, Adding advanced behavioural models in whole building energy simulation: a study on the total energy impact of manual and automated lighting control, Energy Build. 38 (2006) 814-823, http://dx.doi.org/10.1016/j.enbuild.2006.03.002. 
[35] G. Zimmermann, Modeling and simulation of individual user behavior for building performance predictions, in: Proceedings of the 2007 Summer Computer Simulation Conference, San Diego, California, 2007, pp. 913-920.

[36] G.Y. Yun, K. Steemers, Time-dependent occupant behaviour models of window control in summer, Build. Environ. 43 (2008) 1471-1482, http://dx. doi.org/10.1016/j.buildenv.2007.08.001.

[37] J. Tanimoto, A. Hagishima, H. Sagara, Validation of probabilistic methodology for generating actual inhabitants' behavior schedules for accurate prediction of maximum energy requirements, Energy Build. 40 (2008) 316-322, http:// dx doi.org/10.1016/j.enbuild 2007.02.032.

[38] H.B. Rijal, P. Tuohy, J.F. Nicol, M.A. Humphreys, Development of an adaptive window-opening algorithm to predict the thermal comfort, energy use and overheating in buildings, J. Build. Perform. Simul. 1 (2008) 17-30.

[39] J. Page, D. Robinson, N. Morel, J.L. Scartezzini, A generalised stochastic model for the simulation of occupant presence, Energy Build. 40 (2008) 83-98, http://dx.doi.org/10.1016/j.enbuild.2007.01.018.

[40] F. Haldi, D. Robinson, On the behaviour and adaptation of office occupants, Build. Environ. 43 (2008) 2163-2177, http://dx.doi.org/10.1016/j.buildenv. 2008.01.003.

[41] S. Herkel, U. Knapp, J. Pfafferott, Towards a model of user behaviour regarding the manual control of windows in office buildings, Build. Environ. 43 (2008) 588-600, http://dx.doi.org/10.1016/j.buildenv.2006.06.031.

[42] I. Richardson, M. Thomson, D. Infield, A high-resolution domestic building occupancy model for energy demand simulations, Energy Build. 40 (2008) 1560-1566, http://dx.doi.org/10.1016/j.enbuild.2008.02.006.

[43] J. Tanimoto, A. Hagishima, H. Sagara, Validation of methodology for utility demand prediction considering actual variations in inhabitant behaviour schedules, J. Build. Perform. Simul. 1 (2008) 31-42, http://dx.doi.org/10.1080/ 19401490701868471

[44] J. Widén, M. Lundh, I. Vassileva, E. Dahlquist, K. Ellegård, E. Wäckelgård, Constructing load profiles for household electricity and hot water from time-use data-Modelling approach and validation, Energy Build. 41 (2009) 753-768, http://dx.doi.org/10.1016/j.enbuild.2009.02.013.

[45] J. Widén, A.M. Nilsson, E. Wäckelgård, A combined Markov-chain and bottom-up approach to modelling of domestic lighting demand, Energy Build. 41 (2009) 1001-1012, http://dx.doi.org/10.1016/j.enbuild.2009.05.002.

[46] V.L. Erickson, Y. Lin, A. Kamthe, R. Brahme, A. Surana, A.E. Cerpa, et al., Energy efficient building environment control strategies using real-time occupancy measurements, ACM Workshop on Embedded Sensing Systems for Energy-Efficiency in Buildings (2009) 19, http://dx.doi.org/10.1145/1810279. 1810284.

[47] S.C. Gaceo, F.I. Vázquez, J.V. Moreno, Comparison of standard and case-Based user profiles in building's energy performance simulation, Eleventh International IBPSA Conference (2009) 584-590.

[48] M.M. Armstrong, M.C. Swinton, H. Ribberink, I. Beausoleil-Morrison, J. Millette, Synthetically derived profiles for representing occupant-driven electric loads in Canadian housing, J. Build. Perform. Simul. 2 (2009) 15-30, http://dx.doi.org/10.1080/19401490802706653.

[49] J. a. Davis, D.W. Nutter, Occupancy diversity factors for common university building types, Energy Build. 42 (2010) 1543-1551, http://dx.doi.org/10. 1016/j.enbuild.2010.03.025

[50] F. Haldi, D. Robinson, Adaptive actions on shading devices in response to loca visual stimuli, J. Build. Perform. Simul. 3 (2010) 135-153, http://dx.doi.org/10 1080/19401490903580759.

[51] V. Tabak, B. de Vries, Methods for the prediction of intermediate activities by office occupants, Build. Environ. 45 (2010) 1366-1372, http://dx.doi.org/10. 1016/j.buildenv.2009.11.018.

[52] J. Widén, E. Wäckelgård, A high-resolution stochastic model of domestic activity patterns and electricity demand, Appl. Energ. 87 (2010) 1880-1892, http://dx.doi.org/10.1016/j.apenergy.2009.11.006.

[53] E. Azar, C.C. Menassa, A conceptual framework to energy estimation in buildings using agent based modeling, in: B. Johansson, S. Jain, J.

Montoya-Torres, J. Hugan, E. Yuecesan (Eds.), Proceedings of the 2010 Winte Simulation Conference (2010) 3145-3156.

[54] C.J. Andrews, D. Yi, U. Krogmann, J.A. Senick, R.E. Wener, Designing buildings for real occupants: an agent-based approach, IEEE Transactions on Systems, Man, and Cybernetics Part A:Systems and Humans 41 (2011) 1077-1091, http://dx.doi.org/10.1109/TSMCA.2011.2116116.

[55] D. Robinson, U. Wilke, F. Haldi, Multi agent simulation of occupants' presence and behaviour, Proceedings of Building Simulation (2011) 14-16 http://www. ibpsa.org/proceedings/BS2011/P_1671.pdf.

[56] C. Wang, D. Yan, Y. Jiang, A novel approach for building occupancy simulation, Build. Simul. 4 (2011) 149-167, http://dx.doi.org/10.1007/s12273-011-0044-

[57] Y. Yamaguchi, M. Tanaka, Y. Shimoda, Comparison of occupant behavior models applied to a household, in: Proceedings of the Asim2012-1st Asia Conference of International Building Performance Simulation Association, Shanghai, China, 2012

[58] M. Schweiker, F. Haldi, M. Shukuya, D. Robinson, Verification of stochastic models of window opening behaviour for residential buildings, J. Build. Perform. Simul. 5 (2012) 55-74, http://dx.doi.org/10.1080/19401493.2011. 567422 .

[59] C. Liao, Y. Lin, P. Barooah, Agent-based and graphical modelling of building occupancy, J. Build. Perform. Simul. 5 (2012) 5-25, http://dx.doi.org/10.1080/ 19401493.2010.531143.
[60] Y. Zhang, P. Barrett, Factors influencing the occupants' window opening behaviour in a naturally ventilated office building, Build. Environ. 50 (2012) 125-134, http://dx.doi.org/10.1016/j.buildenv.2011.10.018.

[61] U. Wilke, F. Haldi, J.L. Scartezzini, D. Robinson, A bottom-up stochastic model to predict building occupants' time-dependent activities, Build. Environ. 60 (2013) 254-264, http://dx.doi.org/10.1016/j.buildenv.2012.10.021.

[62] R. Andersen, V. Fabi, J. Toftum, S.P. Corgnati, B.W. Olesen, Window opening behaviour modelled from measurements in Danish dwellings, Build. Environ. 69 (2013) 101-113, http://dx.doi.org/10.1016/j.buildenv.2013.07.005.

[63] W.K. Chang, T. Hong, Statistical analysis and modeling of occupancy patterns in open-plan offices using measured lighting-switch data, Build. Simul. 6 (2013) 23-32, http://dx.doi.org/10.1007/s12273-013-0106-y.

[64] D. Aerts, J. Minnen, I. Glorieux, I. Wouters, F. Descamps, A method for the identification and modelling of realistic domestic occupancy sequences for building energy demand simulations and peer comparison, Build. Environ. 75 (2014) 67-78, http://dx.doi.org/10.1016/j.buildenv.2014.01.021.

[65] Y.S. Lee, A.M. Malkawi, Simulating multiple occupant behaviors in buildings: an agent-based modeling approach, Energy Build. 69 (2014) 407-416, http:/ dx.doi.org/10.1016/j.enbuild.2013.11.020.

[66] V. Fabi, S. Maggiora, Occupants ' behaviour in office building: stochastic models for window, 8th Windsor Conference: Counting the Cost of Comfort in Changing World Cumberland Lodge, Windsor, UK, 10-13 April 2014. London : Network for Comfort and Energy Use in Buildings (2014) 10.

[67] T. Buso, S.D. Oca, S.P. Corgnati, The influence of realistic schedules for the use of appliances on the total energy performances in dwellings, (2014) 1-18.

[68] P. Chapman, Coupling multi-agent stochastic simulation of occupants with building simulation, in: Building Simulation and Optimization Conference (BSO14), London, 2014.

[69] A.M. Rysanek, R. Choudhary, DELORES - an open-source tool for stochastic prediction of occupant services demand, J. Build. Perform. Simul. (2014) 37-41, http://dx.doi.org/10.1080/19401493.2014.888595.

[70] H.B. Gunay, W. O’Brien, I. Beausoleil-Morrison, R. Goldstein, S. Breslav, A. Khan, Coupling stochastic occupant models to building performance simulation using the discrete event system specification formalism, J. Build. Perform. Simul. 7 (2014) 1-22, http://dx.doi.org/10.1080/19401493.2013. 866695.

[71] S. D'Oca, V. Fabi, S.P. Corgnati, R.K. Andersen, Effect of thermostat and window opening occupant behavior models on energy use in homes, Build. Simul. 7 (2014) 683-694, http://dx.doi.org/10.1007/s12273-014-0191-6.

[72] J. Langevin, J. Wen, P.L. Gurian, Including occupants in building performance simulation: integration of an agent-based occupant behavior algorithm with Energyplus, in: 2014 ASHRAE/IBPSA-USA, Atlanta, GA, 2014

[73] A. Alfakara, B. Croxford, Using agent-based modelling to simulate occupants' behaviours in response to summer overheating, in: Symposium on Simulation for Architecture and Urban Design, Tampa Florida, USA, 2014

[74] X. Zhou, D. Yan, T. Hong, X. Ren, Data analysis and stochastic modeling of lighting energy use in large office buildings in China, Energy Build. 86 (2015) 275-287, http://dx.doi.org/10.1016/j.enbuild.2014.09.071.

[75] IEA EBC Annex 66, 2014 http://www.annex66.org/.

[76] H.B. Gunay, W. O'Brien, I. Beausoleil-Morrison, Implementation and comparison of existing occupant behaviour models in EnergyPlus, J. Build. Perform. Simul. 1493 (2015) 1-46, http://dx.doi.org/10.1080/19401493.2015. 1102969.

[77] T. Hong, S. D’Oca, W.J.N. Turner, S.C. Taylor-Lange, An ontology to represent energy-related occupant behavior in buildings Part I: Introduction to the DNAs Framework, Build. Environ. (2015), http://dx.doi.org/10.1016/j. buildenv.2015.02.019.

[78] F. Tahmasebi, S. Mostofi, A. Mahdavi, Exploring the implications of different occupancy modelling approaches for building performance simulation results, Energy Procedia 78 (2015) 567-572, http://dx.doi.org/10.1016/j. egypro.2015.11.737.

[79] U.S. Department of Energy (DOE), Commercial reference buildings, (n.d.) http://energy.gov/eere/buildings/commercial-reference-buildings.

[80] F. Tahmasebi, A. Mahdavi, The sensitivity of building performance simulation results to the choice of occupants' presence models: a case study, J. Build. Perform. Simul. 1493 (2015) 1-11, http://dx.doi.org/10.1080/19401493.2015. 1117528.

[81] P.O. Fanger, Thermal Comfort, Danish Technical Press, Copenhagen, Denmark, 1970

[82] I. Richardson, M. Thomson, D. Infield, C. Clifford, Domestic electricity use: a high-resolution energy demand model, Energy Build. 42 (2010) 1878-1887.

[83] No Title, (n.d.). http://www.macmillandictionary.com/dictionary/british/fitfor-purpose.

[84] A. Mahdavi, People in building performance simulation, in: J.L.M. Hensen, R. Lamberts (Eds.), Building Performance Simulation for Design and Operation, Spon Press, 2011, pp. 56-83.

[85] A. Mahdavi, Common fallacies in representation of occupants in building performance simulation, in: Proceedings of Building Simulation Applications 2015-2nd IBPSA-Italy Conference, Bozen-Bolzano University Press, 1-7, 2015.

[86] A. Mahdavi, F. Tahmasebi, The deployment-dependence of occupancy-related models in building performance simulation, Energy Build. (2015), http://dx. doi.org/10.1016/j.enbuild.2015.09.065.

[87] D. Yan, W. O'Brien, T. Hong, X. Feng, H. Burak Gunay, F. Tahmasebi, et al., Occupant behavior modeling for building performance simulation: current state and future challenges, Energy Build. 107 (2015) 264-278, http://dx.doi. org/10.1016/j.enbuild.2015.08.032. 
[88] P. Hoes, J.L.M. Hensen, M.G.L.C. Loomans, B. de Vries, D. Bourgeois, User behavior in whole building simulation, Energy Build. 41 (2009) 295-302, http://dx.doi.org/10.1016/j.enbuild.2008.09.008.

[89] BPIE, Europe's Buildings Under the Microscope, Buildings Performance Institute Europe (BPIE), Brussels, Belgium, 2011.

[90] P. Correia da Silva, V. Leal, M. Andersen, Occupants interaction with electric lighting and shading systems in real single-occupied offices: results from a monitoring campaign, Build. Environ. 64 (2013) 152-168, http://dx.doi.org/ 10.1016/j.buildenv.2013.03.015.

[91] W. O'Brien, H.B. Gunay, The contextual factors contributing to occupants' adaptive comfort behaviors in offices-A review and proposed modeling framework, Build. Environ. 77 (2014) 77-87, http://dx.doi.org/10.1016/j. buildenv.2014.03.024.

[92] U.S. DOE, New Construction - Commercial Reference Buildings (n.d.) http:// energy.gov/eere/buildings/new-construction-commercial-referencebuildings.

[93] H.W. Lin, T. Hong, On variations of space-heating energy use in office buildings, Appl. Energ. 111 (2013) 515-528, http://dx.doi.org/10.1016/j. apenergy.2013.05.040 